MATHEMATICS OF COMPUTATION

Volume 68, Number 226, April 1999, Pages 683-715

S 0025-5718(99)01034-0

\title{
ON BEST POSSIBLE ORDER OF CONVERGENCE ESTIMATES IN THE COLLOCATION METHOD AND GALERKIN'S METHOD FOR SINGULARLY PERTURBED BOUNDARY VALUE PROBLEMS FOR SYSTEMS OF FIRST-ORDER ORDINARY DIFFERENTIAL EQUATIONS
}

\author{
I. A. BLATOV AND V. V. STRYGIN
}

\begin{abstract}
The collocation method and Galerkin method using parabolic splines are considered. Special adaptive meshes whose number of knots is independent of the small parameter of the problem are used. Unimprovable estimates in the $L_{\infty}$-norm are obtained. For the Galerkin method these estimates are quasioptimal, while for the collocation method they are suboptimal.
\end{abstract}

\section{INTRODUCTION}

It is well known that the spline collocation method for a nonstiff boundary value problem leads to a priori high-order accuracy estimates in the uniform norm [1]-[3].

For the Galerkin method in nonstiff problems the corresponding estimates are quasioptimal [4]-[6]. For the investigation of stiff systems it is appropriate to use strongly nonuniform meshes [12]-[15]. This circumstance significantly complicates the problem. Moreover, for stiff problems, it is difficult to select the principal part of a differential operator. To overcome these difficulties, the authors of [7]-[11] proposed Petrov-Galerkin type methods involving special bases in the test spaces; by means of them it may be possible to approximate solutions very well, not only in the center of an interval but also in boundary layers. In the present article we use these ideas. For numerical analysis we use $C^{1}$ quadratic splines on meshes proposed by N. S. Bakhvalov. These meshes have a little number of knots, but they are denser and closer in the boundary layers. This allows us to obtain high-order accuracy with small additional computational work. The estimates obtained in this article have the same accuracy as analogous estimates for nonstiff boundary value problems. It is shown that these estimates are unimprovable, and, for the Galerkin method, they are quasioptimal.

Note that for collocation methods similar ideas are used in the papers by Asher and Weiss [12]-[13] and by Ringhofer [14], but they use other meshes and splines of high defects.

Received by the editor May 28, 1994 and, in revised form, February 11, 1995 and May 26, 1996.

1991 Mathematics Subject Classification. Primary 65-02, 65L99; Secondary 65G99, 45A10.

Key words and phrases. Finite element method for singular perturbation problems, collocation method, Galerkin method.

(C)1999 American Mathematical Society 
In conclusion we note that our investigation of the Galerkin method is based on the remarkable ideas of J. A. Nitsche, F. Natterer, R. Scott, A. H. Schatz and L. B. Wahlbin [15]-[18].

\section{Statement of the problem. \\ PRELININARIES AND NOTATION}

Let $\Delta:-1=t_{-p}<\cdots<t_{p}=1$ denote any partition of the interval $[-1,1]$, and let $h_{s}=t_{s+1}-t_{s}$. By $B_{i, r}(t)$ we denote the $B$-spline of degree $r$ on the partition $\Delta$ having support $\left[t_{i}, t_{i+r+1}\right]$. We assume that $B_{i, r}$ are normalised so that $\sum_{j=i-r}^{i} B_{j, r}(t) \equiv 1, t \in\left[t_{i}, t_{i+1}\right]$. Let $S(\Delta, r, 1)$ be the space of polynomial splines of degree $r$ and defect 1 on the partition $\Delta$. Throughout this paper $\varepsilon$ denotes a small positive parameter; $C, C_{1}, C_{2} \cdots$ will be used to denote positive constants independent of $\varepsilon$ and the partition $\Delta$.

As usual, $C^{s}[-1,1]$ denotes the space of all scalar, vector or matrix functions on $[-1,1]$ which are continuous together with their derivatives up through order $s$ in $[-1,1] ;\|\cdot\|_{C^{s}}$ will be the norm in this space.

We use the notation $\|\cdot\|_{p}$ for the norm in $L_{p}[-1,1](1 \leq p \leq \infty)$. Using the sharp order function, we shall write $f(\varepsilon, m)=O^{*}(g(\varepsilon, m))$ if there exist constants $C_{1}$ and $C_{2}$ such that, for some $\varepsilon_{0}>0$ and $m_{0} \in N$, and for every $\varepsilon \in\left(0, \varepsilon_{0}\right]$ and $m \geq m_{0}$, the estimates

$$
C_{1}|f(\varepsilon, m)| \leq|g(\varepsilon, m)| \leq C_{2}|f(\varepsilon, m)|
$$

hold.

If only the first inequality holds, we shall write $f(\varepsilon, m)=O(g(\varepsilon, m))$.

On the interval $[-1,1]$ consider now the problem

$$
\begin{gathered}
L_{\varepsilon} x=\varepsilon x^{\prime}-A(t) x=d(t), \quad x=\left(x^{1}, x^{2}, \ldots, x^{n}\right)^{T} \in R^{n}, \\
x^{1}(-1)=\cdots=x^{k}(-1)=x^{k+1}(1)=\cdots=x^{n}(1)=0 .
\end{gathered}
$$

Here $A(t)$ is a matrix and $d(t)$ is a vector function of class $C^{3}$. Suppose that the matrix $A(t)$ has eigenvalues $\nu_{1}(t), \nu_{2}(t), \ldots, \nu_{n}(t)$ such that, for any $t \in[-1,1]$, $\nu_{1}(t)<\nu_{2}(t)<\cdots<\nu_{k}(t)<0<\nu_{k+1}(t)<\cdots<\nu_{n}(t) ;\left|\nu_{i}(t)\right| \geq \nu_{0}>0$.

Let the matrix $B$ reduce the matrix $A(t)$ to diagonal form, i.e.,

$$
B^{-1} A B=\operatorname{diag}\left(\nu_{1}(t), \nu_{2}(t), \ldots, \nu_{n}(t)\right) .
$$

Represent the matrix $B$ in the corresponding block form

$$
B=\left\|\begin{array}{ll}
B_{11} & B_{12} \\
B_{21} & B_{22}
\end{array}\right\|
$$

where $B_{11}$ is a $k \times k$ matrix.

Suppose that $\operatorname{det} B_{11}(-1) \operatorname{det} B_{11}(1) \operatorname{det} B_{22}(-1) \operatorname{det} B_{22}(1) \neq 0$.

The following statements are known [19].

Lemma 1.1. There exists a constant $C$ and an $\varepsilon_{0}>0$ such that for all $\varepsilon \in\left(0, \varepsilon_{0}\right]$ the operator $L_{\varepsilon}$ has a Green function $G_{\varepsilon}(t, \xi)$ satisfying

$$
\left\|\frac{\partial^{i}}{\partial \xi^{i}} G_{\varepsilon}(t, \xi)\right\| \leq C / \varepsilon^{i+1} \exp \left(-\nu_{0}|t-\xi| / \varepsilon\right)
$$

and, for the integral operator associated with $G_{\varepsilon}(t, \xi)$, the following estimates hold:

$$
\left\|G_{\varepsilon}\right\|_{C \rightarrow C} \leq C, \quad\left\|G_{\varepsilon}\right\|_{C \rightarrow C^{1}} \leq C / \varepsilon
$$


Lemma 1.2. There exists an $\varepsilon_{0}>0$ such that for all $\varepsilon \in\left(0, \varepsilon_{0}\right]$ the problem (1.1)(1.2) has a unique solution $x_{\varepsilon}(t)$. Moreover, for $i=0,1,2,3$, we have

$$
\left\|x_{\varepsilon}^{(i)}(t)\right\| \leq C\left(1+\varepsilon^{-k}\left(\exp \left(\nu_{0}(t-1) / \varepsilon\right)+\exp \left(\nu_{0}(-1-t) / \varepsilon\right)\right)\right) .
$$

Lemma 1.3. The homogeneous system $L_{\varepsilon} x=0$ has the fundamental system of solutions (F.S.S.) $\zeta_{1}(t, \varepsilon), \ldots, \zeta_{n}(t, \varepsilon)\left(0<\varepsilon \leq \varepsilon_{0}\right)$ for which the representations

$$
\zeta_{i}(t, \varepsilon)=\left\{b_{i}(t)+\varepsilon \chi_{i}(t, \varepsilon)\right\} \exp \left(\frac{1}{\varepsilon} \int_{-1}^{t} \nu_{i}(s) d s\right), \quad i=1,2, \ldots, k,
$$

$$
\zeta_{i}(t, \varepsilon)=\left\{b_{i}(t)+\varepsilon \chi_{i}(t, \varepsilon)\right\} \exp \left(-\frac{1}{\varepsilon} \int_{t}^{1} \nu_{i}(s) d s\right), \quad i=k+1, k+2, \ldots, n,
$$

are valid. Here

$$
\left\|\chi_{i}^{(j)}(t, \varepsilon)\right\|_{\infty} \leq C / \varepsilon^{j}, \quad j=0,1,2,3
$$

and $b_{i}(t)$ is an eigenvector of $A(t)$ associated with the eigenvalue $\nu_{i}(t)$.

\section{Galerkin AND COLLOCATiOn METHOds. FORMULATION OF THE MAIN RESULTS}

To construct a suitable partition of $[-1,1]$ we use the Bakhvalov approach [20]. Let $a=1-\left(3 / \nu_{0}\right) \varepsilon \ln (1 / \varepsilon)$; note that $a \rightarrow 1$ as $\varepsilon \rightarrow 0$. Define

$$
g(t)=\left\{\begin{array}{l}
t, \quad t \in[0, a], \\
a-3 \varepsilon / \nu_{0}+\left(3 / \nu_{0}\right) \exp \left(\left(\nu_{0} /(3 \varepsilon)\right)(t-1)\right), \quad t \in[a, 1] .
\end{array}\right.
$$

For $t \in[-1,0]$ we set $g(t)=-g(-t)$. Then $g(t)$ belongs to $C^{1}[-1,1]$ and maps $[-1,1]$ onto $[-b, b]$ in a one-to-one fashion, where $b=a+\left(3 / \nu_{0}\right)(1-\varepsilon)$. Let $m$ be any natural number. On $[0, b]$ we set

$$
\tau_{i}=\left\{\begin{array}{l}
a i / m, \quad i=0,1, \ldots, m \\
a+(b-a)(i-m) / m, \quad i=m+1, m+2, \ldots, 2 m .
\end{array}\right.
$$

Points $\tau_{i}$ on the interval $[-b, 0]$ are introduced symmetrically.

Knots $t_{i}$ of the partition $\Delta$ of the interval $[-1,1]$ will be defined by $t_{i}=g^{-1}\left(\tau_{i}\right)$, where $g^{-1}$ is the inverse of the function $g$. Let $h=1 / m$. We shall distinguish three cases:

$$
\begin{aligned}
& h \ll \varepsilon, \\
& 0<C_{1} \leq \varepsilon / h \leq C_{2}, \\
& \varepsilon \ln (1 / \varepsilon) \ll h .
\end{aligned}
$$

Recall here that $h_{j}=t_{j+1}-t_{j}$.

Lemma 2.1. In the case $(\gamma)$ the following relations hold:

$$
\begin{gathered}
3 \varepsilon /\left(\nu_{0}(j-m+2)\right) \leq h_{j} \leq 3 \varepsilon /\left(\nu_{0}(j-m)\right), \quad j>m+1, \\
h_{j}=O(\varepsilon \ln (1 / \varepsilon)), \quad j=m, \\
h_{j}=h(1+O(\varepsilon \ln (1 / \varepsilon))), \quad j=0,1, \ldots, m-1 .
\end{gathered}
$$


Proof. For $j \geq m$ we have

$$
\begin{aligned}
& h_{j}= g^{-1}\left(\tau_{j+1}\right)-g^{-1}\left(\tau_{j}\right) \\
&=3 \varepsilon / \nu_{0}\left\{\ln \left[(j-m+1)(b-a) / m+3 \varepsilon / \nu_{0}\right]\right. \\
&\left.\quad \quad-\ln \left[(j-m)(b-a) / m+3 \varepsilon / \nu_{0}\right]\right\} .
\end{aligned}
$$

Hence, in accordance with MacLaurin's formula, we get (2.1) for $j \geq m+1$. For $j \leq m$ these formulas are obvious. The lemma is proved.

We next define trial and test spaces. For the trial spaces we take

$$
\begin{array}{r}
E=\left\{u=\left(u^{1}(t), u^{2}(t), \ldots, u^{n}(t)\right)^{T}, u^{i}(t) \in S(\Delta, 2,1), u^{1}(-1)=u^{2}(-1)\right. \\
\left.=\cdots=u^{k}(-1)=u^{k+1}(1)=\cdots=u^{n}(1)=0\right\} .
\end{array}
$$

For the test space consider $F=L_{\varepsilon} E$. It is clear that $\operatorname{dim} E=\operatorname{dim} F=(4 m+1) n$, independently of $\varepsilon$.

Define the collocation method in the following manner. First introduce collocation points

$$
\begin{aligned}
& \xi_{i}=\left(t_{i-1}+t_{i}\right) / 2, \quad i=1,2, \ldots, m, \\
& \xi_{i}=\left(t_{i}+t_{i+1}\right) / 2, \quad i=-1,-2, \ldots,-m, \\
& \xi_{i}=t_{i-1}, \quad i=m+1, m+2, \ldots, 2 m+1, \\
& \xi_{i}=t_{i+1}, \quad i=-m-1,-m-2, \ldots,-2 m-1 .
\end{aligned}
$$

Let $I=\{-2 m-1,-2 m, \ldots,-m-3,-m-1,-m, \ldots,-1,1,2, \ldots, m, m+1$, $m+3, m+4, \ldots, 2 m+1\}$ be the index set. The collocation method consists in finding $u(t) \in E$ so that $u(t)$ satisfies

$$
\begin{aligned}
\left.L_{\varepsilon} u\right|_{t=\xi_{j}} & =d\left(\xi_{j}\right), \quad j \in I \\
\left\{\left.L_{\varepsilon} u\right|_{t=\xi_{m+2}}\right\}^{\nu} & =\left\{d\left(\xi_{m+2}\right)\right\}^{\nu}, \quad \nu=1,2, \ldots, k, \\
\left\{\left.L_{\varepsilon} u\right|_{t=\xi_{-m-2}}\right\}^{\nu} & =\left\{d\left(\xi_{-m-2}\right)\right\}^{\nu}, \quad \nu=k+1, \ldots, n
\end{aligned}
$$

in case $(\alpha)$, and $u(t)$ satisfies

$$
\left.L_{\varepsilon} u\right|_{t=t_{j}}=d\left(t_{j}\right), \quad j=-2 m,-2 m+1, \ldots, 2 m
$$

in cases $(\beta)$ and $(\gamma)$.

The Galerkin method of least-square type consists in finding $u(t) \in E$ so that for each $v \in F$,

$$
\left(L_{\varepsilon} u, v\right)=(d, v) .
$$

Here $(,$,$) denotes the inner product in \left(L_{2}[-1,1]\right)^{n}$.

In this paper the Galerkin method is considered only in the case $(\gamma)$. Recall that $h=1 / m$.

Theorem 1. There exist constants $C>0, \varepsilon_{0}>0, h_{0}>0, \gamma_{0}>0$ such that for all $\varepsilon \in\left(0, \varepsilon_{0}\right]$ and $h \in\left(0, h_{0}\right]$ with $h \leq \gamma_{0} \varepsilon$ the problem (2.6) has a unique solution $u(t)$ and

$$
\left\|x_{\varepsilon}(t)-u(t)\right\|_{\infty}+\varepsilon\left\|x_{\varepsilon}^{\prime}(t)-u^{\prime}(t)\right\|_{\infty} \leq C h^{2} .
$$

Theorem 2. For every $\gamma_{1}>0$ there exist numbers $C>0, \varepsilon_{0}>0, h_{0}>0$ such that for all $\varepsilon \in\left(0, \varepsilon_{0}\right]$ and $h \in\left(0, h_{0}\right]$ with $\gamma_{1} \varepsilon \leq h \leq \varepsilon / \gamma_{1}$ the problem (2.6) has a unique solution $u(t)$ and the estimate (2.8) holds. 
Theorem 3. There exist numbers $\varepsilon_{0}>0, h_{0}>0, \gamma_{2}>0, C>0$ such that for all $\varepsilon \in\left(0, \varepsilon_{0}\right]$ and $h \in\left(0, h_{0}\right]$ with $\varepsilon \ln (1 / \varepsilon) \leq \gamma_{2}$ h the problem $(2.3)-(2.5)$ has a unique solution $u(t)$, and the estimate (2.8) is valid.

The following theorem shows that, for the collocation method, the estimates (2.8) are best possible in order.

Theorem 4. There exist a constant $C_{1}>0$ and a function $d(t) \in C^{3}[-1,1]$ independent of $\varepsilon$ and $h$ such that, for sufficiently small $\varepsilon$ and $h$, in all three cases $(\alpha)$, $(\beta),(\gamma)$ the estimate

$$
\left\|x_{\varepsilon}(t)-u(t)\right\|_{\infty} \geq C_{1} h^{2}
$$

holds.

Theorem 5. There exist numbers $\varepsilon_{0}>0, h_{0}>0, \gamma_{2}>0, C>0$ such that for all $\varepsilon \in\left(0, \varepsilon_{0}\right]$ and $h \in\left(0, h_{0}\right]$ satisfying $\varepsilon \ln (1 / \varepsilon) \leq \gamma_{2} h$ the problem $(2.7)$ has a unique solution $u(t)$, and

$$
\left\|x_{\varepsilon}(t)-u(t)\right\|_{\infty} \leq C h^{3} .
$$

Remark 2.1. As will be shown

$$
\inf _{u \in E}\left\|x_{\varepsilon}(t)-u(t)\right\|_{\infty}=O\left(h^{3}\right) .
$$

Thus the estimate (2.10) in general is unimprovable and quasioptimal.

\section{Proof of Theorems $1-3$}

The proofs of Theorems 1-3 are based on the notion of interpolation projection.

Definition 3.1. The linear operator $P: C[-1,1] \rightarrow F=L_{\varepsilon} F$ such that $P P=P$ and $(P d)\left(t_{j}\right)=d\left(t_{j}\right)$ for any $d \in C[-1,1](j=-2 m, \ldots, 2 m)$ is said to be the interpolation projection for cases $(\alpha)$ and $(\beta)$; in the case $(\gamma)$ the interpolation conditions take the form

$$
\begin{gathered}
(P d)\left(\xi_{j}\right)=d\left(\xi_{j}\right) \quad \text { for } j \in I, \\
\left\{(P d)\left(\xi_{m+2}\right)\right\}^{\nu}=\left\{d\left(\xi_{m+2}\right)\right\}^{\nu}, \quad \nu=1, \ldots, k, \\
\left\{(P d)\left(\xi_{-m-2}\right)\right\}^{\nu}=\left\{d\left(\xi_{-m-2}\right)\right\}^{\nu}, \quad \nu=k+1, \ldots, n .
\end{gathered}
$$

Lemma 3.1. There exist numbers $\varepsilon_{0}>0, h_{0}>0 . \gamma_{2}>0, C>0$ such that for all $\varepsilon \in\left(0, \varepsilon_{0}\right]$ and $h \in\left(0, h_{0}\right]$, if $\varepsilon \ln (1 / \varepsilon) \leq \gamma_{2} h$, then the interpolation projection $P(\varepsilon, h)$ exists and

$$
\|P(\varepsilon, h)\|_{C \rightarrow C} \leq C .
$$

Remark 3.1. Analogous statements are true in the cases $(\alpha)$ and $(\beta)$.

Lemma 3.2. Let $\hat{d}(t) \in F$ be the best approximation of $d(t) \in F$ in the sense of the norm in $C[-1,1]$. Then in cases $(\alpha),(\beta)$ and $(\gamma)$ we have $\|d-\hat{d}\|_{\infty} \leq C h^{2}$ for some $C$.

Lemma 3.3. For all $\varepsilon \in\left(0, \varepsilon_{0}\right]$ and $h \in\left(0, h_{0}\right]$ such that the interpolation projection $P$ exists, the collocation problem has a solution $u(t)$ in cases $(\alpha),(\beta)$ and $(\gamma)$. Moreover, for $i=0,1$,

$$
\left\|x_{\varepsilon}(t)-u(t)\right\|_{C^{i}} \leq\left\|G_{\varepsilon}\right\|_{C \rightarrow C^{i}}\left(1+\|P\|_{C \rightarrow C}\right)\|d-\hat{d}\|_{C} .
$$


It is easy to see that Lemmas $3.1-3.3$ and Lemma 1.3 imply Theorems 1-3.

We outline the proofs of Lemmas 3.1-3.3. The proof of Lemma 3.3 follows from the estimates

$$
\begin{aligned}
\left\|x_{\varepsilon}-u\right\|_{C^{i}} & =\left\|G_{\varepsilon} P d-G_{\varepsilon} d\right\|_{C^{i}} \leq\left\|G_{\varepsilon}\right\|_{C \rightarrow C^{i}}\|P d-d\|_{C} \\
& \leq\left\|G_{\varepsilon}\right\|_{C \rightarrow C^{i}}\left(\|P(d-\hat{d})\|+\|d-\hat{d}\|_{C}\right)
\end{aligned}
$$

and Lemmas 3.1 and 3.2 .

The proof of Lemma 3.2 is based on the approximation theorems of de Boor [21] for the space of splines on nonuniform meshes. These theorems and Lemmas 1.2 and 2.1 imply that there is a function $\stackrel{*}{x}(t) \in E$ which satisfies

$$
\left\|x_{\varepsilon}(t)-\stackrel{*}{x}(t)\right\|_{\infty} \leq C g^{3}, \quad \varepsilon\left\|x_{\varepsilon}^{\prime}(t)-\stackrel{*}{x}^{\prime}(t)\right\|_{\infty} \leq C h^{2} .
$$

Letting $\stackrel{*}{d}=L_{\varepsilon} \stackrel{*}{x}$, due to (3.1) we obtain $\|d-\hat{d}\|_{\infty} \leq\|d-\stackrel{*}{d}\|_{\infty} \leq C h^{2}$, i.e., the assertion of Lemma 3.2. For details of the proof, see [7] and [9].

Remark 3.2. The case $a=1-2 / \nu_{0} \varepsilon \ln (1 / \varepsilon)$ was considered in [7]-[9]. To establish the statements in the case $a=1-3 / \nu_{0} \varepsilon \ln (1 / \varepsilon)$ there are no essential changes.

The proof of Lemma 3.1 is based on the following statements.

Proposition 3.1. The interpolation projection $P$ exists if and only if there exists a basis $N_{1}, \ldots, N_{q}\left(N_{i}=N_{i}(t, \varepsilon, h)\right)$ in $F$ such that, in the cases $(\alpha)$ and $(\beta)$, the system of linear equations

$$
\sum_{j=1}^{q} \alpha_{j} N_{j}\left(t_{i}\right)=f_{i}, \quad i=-2 m, \ldots, 2 m
$$

has a unique solution; and, in the case $(\gamma)$, the system of linear equations

$$
\begin{gathered}
\sum_{j=1}^{q} \alpha_{j} N_{j}\left(\xi_{i}\right)=f_{i}, \quad i \in I, \\
\left\{\sum_{j=1}^{q} \alpha_{j} N_{j}\left(\xi_{m+2}\right)\right\}^{\nu}=\left\{f_{m+2}\right\}^{\nu}, \quad \nu=1,2, \ldots, k, \\
\left\{\sum_{j=1}^{q} \alpha_{j} N_{j}\left(\xi_{-m-2}\right)\right\}^{\nu}=\left\{f_{-m-2}\right\}^{\nu}, \quad \nu=k+1, \ldots, n,
\end{gathered}
$$

has a unique solution for any collection of vectors $f^{j} \in R^{n}$.

Proposition 3.2. Let the basis $\left\{N_{j}(t)\right\}$ from Proposition 3.1 satisfy the following conditions:

1) $\sum_{j=1}^{q}\left\|N_{j}(t)\right\|_{R^{n}} \leq C_{1}$ for any $t \in[-1,1]$.

2) There exists a constant $C_{2}$ such that for any collection of $n$-dimensional vectors $f_{j}$ with $\left\|f_{j}\right\|_{R^{n}} \leq 1$, the solution of system (3.2) (or (3.3)) satisfies the estimate $\max _{1 \leq j \leq q}\left|\alpha_{j}\right| \leq C_{2}$.

Then for the family of interpolation projections, the uniform estimate $\|P\|_{C \rightarrow C} \leq$ $C_{1} C_{2}$ holds. 
For proofs of these simple assertions, see [8] and [9].

A basis satisfying the conditions in Propositions 3.1 and 3.2 is called an $N$-basis. Thus to prove Lemma 3.1 and Theorems $1-3$ it is sufficient to construct an $N$-basis in $F=F(\varepsilon, h)$.

Lemma 3.4. There are functions

$$
\begin{aligned}
N_{i j}(t, \varepsilon) & =b_{j}\left(t_{i+1}\right) B_{i+1,1}(t)+\Phi_{i j}(t, \varepsilon)+\mu_{i j}(t, \varepsilon), \\
(i & =m+i_{0}, m+i_{0}+1, \ldots, 2 m-2 ; \\
i & \left.=-2 m-2, \ldots,-m-i_{0}-4 ; j=k+1, k+2, \ldots, n\right), \\
(i & =m+i_{0}-1, \ldots, 2 m-2 ; \\
i & \left.=-2 m-2, \ldots,-m-i_{0}-3 ; j=1,2, \ldots, k\right),
\end{aligned}
$$

in the space $F$ such that

$$
\begin{gathered}
\operatorname{supp} \mu_{i j} \subset\left[t_{-2 m}, t_{-m-1}\right] \cup\left[t_{m+1}, t_{2 m}\right], \\
\operatorname{supp} \Phi_{i j}(t, \varepsilon) \subset\left[t_{i+1}, t_{i+3}\right], \\
\left\|d^{\nu} \Phi_{i j} / d t^{\nu}\right\|_{L^{\infty}[-1,1]} \leq C /(|j|-m) h_{j}^{-\nu}, \quad \nu=0,1, \\
\left\|d^{\nu} \mu_{i j} / d t^{\nu}\right\|_{L^{\infty}\left[t_{q}, t_{q+1}\right]} \leq C / \max \left\{(|i|-m)^{3},(|q|-m)^{3}\right\} h_{q}^{-\nu}, \quad \nu=0,1,
\end{gathered}
$$

where $i_{0}$ is a sufficiently large number and $C$ is independent of $i_{0}$.

Lemma 3.4 was proved in [7] and [11], where the estimates (3.7) and (3.8) were obtained only for $\nu=0$. We shall prove them for $\nu=1$. As it was shown in the proof of a lemma on basis functions in [9], the functions $\mu_{i j}(t, \varepsilon)$ can be written in the form $\mu_{i j}(t, \varepsilon)=L_{\varepsilon} \kappa_{i j}(t, \varepsilon)$, where $\kappa_{i j} \in[S(\Delta, 2,1)]^{n}$, and, moreover,

$$
\left\|\kappa_{i j}\right\|_{\infty} \leq C /(|i|-m) \text {. }
$$

Consider the function $\mu_{i j}(t, \varepsilon)$ on an arbitrary interval $\left[t_{q}, t_{q+1}\right]$. Represent this function in the form

$$
\mu_{i j}(t, \varepsilon)=\varepsilon x^{\prime}(t, \varepsilon)-A_{q}(t) \kappa_{i j}(t, \varepsilon)-\left(A(t)-A_{q}(t)\right) \kappa_{i j}(t, \varepsilon),
$$

where

$$
A_{q}(t)=A\left(t_{q}\right)+A^{\prime}\left(t_{q}\right)\left(t-t_{q}\right)+\frac{1}{2} A^{\prime \prime}\left(t_{q}\right)\left(t-t_{q}\right)^{2} .
$$

Taking into account the smoothness of $A(t)$ and the fact that $\kappa_{i j}(t, \varepsilon)$ is a polynomial of the second degree on $\left[t_{q}, t_{q+1}\right]$, by (3.9) and Lemma 2.1 we have

$$
\begin{aligned}
& \left\|\frac{d^{\nu}}{d t^{\nu}}\left(A(t)-A\left(t_{q}\right)\right) \kappa_{i j}(t, \varepsilon)\right\|_{L_{\infty}\left[t_{q}, t_{q+1}\right]} \leq C h_{q}^{2} /(|i|-m) \\
& \quad \leq C \varepsilon^{2} /((|i|-m)(|q|-m)) \leq C / \max \left\{(|i|-m)^{3},(|q|-m)^{3}\right\}
\end{aligned}
$$

for $\nu=0,1$. According to estimates (3.11) and (3.8), for $\nu=0$ we have

$$
\left\|\varepsilon x^{\prime}(t, \varepsilon)-A_{q}(t) \kappa_{i j}(t, \varepsilon)\right\|_{L_{\infty}\left[t_{q}, t_{q+1}\right]} \leq C / \max \left\{(|i|-m)^{3},(|q|-m)^{3}\right\} .
$$

The function $\varepsilon \kappa_{i j}(t, \varepsilon)-A_{q}(t) \kappa_{i j}(t, \varepsilon)$ is an $n$-dimensional vector function, each component of which is a polynomial of degree $\leq 4$ on $\left[t_{q}, t_{q+1}\right]$. Using the equivalence of norms in the space of polynomials of fourth degree on $\left[t_{q}, t_{\dot{q}+1}\right]$, by $(3.12)$ 
we get

$$
\left\|\frac{d}{d t}\left(\varepsilon \kappa_{i j}^{\prime}-A_{q}(t) \kappa_{i j}\right)\right\|_{L_{\infty}\left[t_{q}, t_{q+1}\right]} \leq C h_{q}^{-1} / \max \left\{(|i|-m)^{3},(|q|-m)^{3}\right\} .
$$

By (3.10), (3.12) and (3.13) the estimate (3.8) follows from $\nu=1$. The estimate (3.7) for $\nu=1$ may be proved analogously. The lemma is proved.

For $i=-m-i_{0}-2, \ldots, m+i_{0}-2$ and $j=1, \ldots, k$, put

$$
N_{i j}(t)=L_{\varepsilon}\left(B_{i, 2} b_{j}\left(t_{i}\right)\right),
$$

and for $i=-m-i_{0}-3, \ldots, m+i_{0}-1$ and $j=k+1, \ldots, n$ define

$$
N_{i j}(t)=L_{\varepsilon}\left(B_{i+1,2} b_{j}\left(t_{i+1}\right)\right) \text {. }
$$

As was shown in [8] and [11], the set $\left\{N_{i j}\right\}$ is a basis in $F$ in cases $(\beta)$ and $(\gamma)$. From (3.9) and (3.8) it follows that it is an $N$-basis. This completes the proof of Theorem 2.3.

Note that in the case $(\alpha)$ (Theorem 1) the proof of Lemma 3.1 is considerably easier and does not require construction of an $N$-basis (see [9]).

\section{Proof of Theorem 4}

Let $b_{1}(t)=\left(b_{1,1}(t), \ldots, b_{1, n}(t)\right)$ be an eigenvector of the matrix $A(t)$, associated with $\lambda_{1}(t)$, and let $e_{i}=(0, \ldots, 0,1,0, \ldots, 0)$ be the unit vector. Put

$$
d(t)=A(t) \sum_{i=1}^{n}\left(b_{1, i}(-1) \frac{t-1}{2}-b_{1, i}(1) \frac{t+1}{2}\right) e_{i} .
$$

Let $x_{\varepsilon}$ be the solution of the problem (1.1)-(1.2), and $u(t)$ the unique solution of the corresponding collocation problem (2.6). We shall prove that the estimate (2.9) holds (in the cases $(\alpha),(\beta),(\gamma)$ ), if $\varepsilon$ and $h$ are sufficiently small.

Let

$$
\bar{x}(t)=\sum_{i=1}^{n}\left(\zeta_{1, i}(-1, \varepsilon) \frac{t-1}{2}-\zeta_{1, i}(1, \varepsilon) \frac{t+1}{2}\right) e_{i}
$$

and

$$
\bar{d}(t)=L_{\varepsilon} x(t)
$$

where $\zeta_{1}(t, \varepsilon)=\left(\zeta_{1,1}(t, \varepsilon), \ldots, \zeta_{1, n}(t, \varepsilon)\right)^{T}$ is an element of an F.S.S. of the equation $L_{\varepsilon} x=0$ (see (1.6)). From (1.6)-(1.8), (4.1) and (4.3) we conclude that

$$
\|d(t)-\bar{d}(t)\|_{C^{3}} \leq C \varepsilon
$$

Let $x_{\varepsilon}(t)=G_{\varepsilon} d(t)$ and $\bar{x}_{\varepsilon}(t)=G_{\varepsilon} \bar{d}(t)$ be solutions of the problem (1.1)-(1.2) with right-hand sides $d(t)$ and $\bar{d}(t)$, respectively, and let $u(t)$ and $\bar{u}(t)$ be solutions of the corresponding collocation problem in cases $(\alpha),(\beta),(\gamma)$. Take $y_{\varepsilon}(t)=$ $\bar{x}_{\varepsilon}(t)-x_{\varepsilon}(t)$ and $v(t)=\bar{u}(t)-u(t)$.

Proposition 4.1. For sufficiently small $\varepsilon$ and $h$

$$
\left\|y_{\varepsilon}(t)-v(t)\right\|_{\infty} \leq C \varepsilon h^{2}
$$


Indeed, $y_{\varepsilon}(t)$ solves the problem (1.1)-(1.2) with the right-hand side $\bar{d}(t)-d(t)$, and $v(t)$ solves the corresponding collocation problem. Then the estimate (4.5) follows from (4.4) and Theorems 1-3. The proposition is proved.

Rewrite the estimate (4.5) in the form

$$
\left\|\left(\bar{x}_{\varepsilon}-\bar{u}\right)-\left(x_{\varepsilon}-u\right)\right\|_{\infty} \leq C \varepsilon h^{2} .
$$

This means that the estimate (2.9) will be proved when we show that

$$
\left\|\bar{x}_{\varepsilon}(t)-\bar{u}(t)\right\|_{\infty} \geq C h^{2} .
$$

From (4.2) and (4.3) it follows that

$$
\bar{x}_{\varepsilon}(t)=\bar{x}(t)+\zeta_{1}(t, \varepsilon),
$$

since $\bar{x}_{\varepsilon}(-1)=\bar{x}_{\varepsilon}(1)=0$. Let us prove the estimate (4.7). First of all we observe that, due to (1.6)-(1.8), we have

$$
\begin{aligned}
\frac{d^{2}}{d t^{2}}\left(\zeta_{1}(t, \varepsilon)\right)=\left(\frac{\nu_{1}(t)}{\varepsilon}\right)^{2} & b_{1}(t) \exp \left\{\frac{1}{\varepsilon} \int_{-1}^{t} \nu_{1}(s) d s\right\} \\
+ & O\left(\varepsilon^{-1}\right) \exp \left\{\frac{1}{\varepsilon} \int_{-1}^{t} \nu_{1}(s) d s\right\} .
\end{aligned}
$$

Since for $-1 \leq t \leq t_{m-2}$ collocation points coincide with knots of the partition $\Delta$ in all three cases, we have $\varepsilon \bar{u}\left(t_{i}\right)=A\left(t_{i}\right) \bar{u}\left(t_{i}\right)+d\left(t_{i}\right)(i=-2 m,-2 m+1, \ldots, m-3)$. Hence on $\left[-1, t_{-m-3}\right]$,

$$
\varepsilon \bar{u}^{\prime}(t)=R[A(t) \bar{u}(t)+d(t)],
$$

where $R$ denotes the projection which maps every $n$-dimensional vector-function $f$ into the $n$-dimensional broken line interpolating $f$ on $t_{-2 m}, \ldots, t_{-m-3}$. From (4.10) we have

$$
\bar{u}(t)=\bar{u}(-1)+\frac{1}{\varepsilon} \int_{-1}^{t} R[A(s) \bar{u}(s)+\bar{d}(s)] d s
$$

for $t \in\left[-1, t_{-m-3}\right]$. It is obvious that

$$
\bar{x}_{\varepsilon}(t)=\bar{x}_{\varepsilon}(-1)+\frac{1}{\varepsilon} \int_{-1}^{t}\left[A(s) x_{\varepsilon}(s)+\bar{d}(s)\right] d s .
$$

From (4.11) and (4.12) we obtain

$$
\begin{aligned}
\bar{x}_{\varepsilon}(t)-\bar{u}(t)=\left(\bar{x}_{\varepsilon}(-1)-\bar{u}(-1)\right)+\frac{1}{\varepsilon} \int_{-1}^{t}(\bar{d}(s)-R \bar{d}(s)) d s \\
+\frac{1}{\varepsilon} \int_{-1}^{t} R A(s)\left(\bar{x}_{\varepsilon}(s)-\bar{u}(s)\right) d s=S_{1}+S_{2}+S_{3} .
\end{aligned}
$$

Assume that the estimate (4.7) does not hold. Then there exists a function $\nu(\varepsilon, h) \rightarrow 0$ as $\varepsilon \rightarrow 0, h \rightarrow 0$ such that

$$
\left\|\bar{x}_{\varepsilon}(t)-\bar{u}(t)\right\|_{\infty} \leq \nu(\varepsilon, h) h^{2} .
$$

To be specific, let $m$ be an even number. Put $t=t_{-3 / 2 m}$ in (4.13). Then from (4.14) we have

$$
\left\|\bar{x}_{\varepsilon}(-1)-\bar{u}(-1)\right\|_{R^{n}} \leq \nu(\varepsilon, h) h^{2}
$$


and

$$
\begin{aligned}
& \left\|\frac{1}{\varepsilon} \int_{-1}^{t_{-3 / 2 m}} R A(s)\left[\bar{x}_{\varepsilon}(s)-\bar{u}(s)\right] d s\right\|_{R^{n}} \\
& \quad \leq C\left(t_{-3 / 2 m}+1\right) / \varepsilon \nu(\varepsilon, h) h^{2} \leq C \nu(\varepsilon, h) h^{2} .
\end{aligned}
$$

The last inequality holds since Lemma 2.1 implies that, for $-2 m \leq i \leq-3 / 2 m$ in cases $(\alpha),(\beta),(\gamma)$,

$$
C_{1} \varepsilon h \leq t_{i+1}-t_{i} \leq C_{2} \varepsilon h, \quad C_{1}>0, C_{2}>0 .
$$

Hence $\left(t_{-3 / 2 m}+1\right) \leq C \varepsilon$.

Further, due to the smoothness of $\bar{d}(t)$ and (4.17),

$$
\left\|\frac{1}{\varepsilon} \int_{-1}^{t_{-3 m / 2}}(\bar{d}(s)-R \bar{d}(s)) d s\right\|_{R^{n}} \leq C \varepsilon^{-1}\left(t_{-3 m / 2}+1\right)(\varepsilon h)^{2} \leq C(\varepsilon h)^{2} .
$$

Let $I$ be the unit matrix and $E$ be the identity operator in $C[-1,1]$. Then, by (1.6) and (4.8),

$$
\begin{aligned}
\frac{1}{\varepsilon} \int_{-1}^{t_{-3 m / 2}}\left[A(s) \bar{x}_{\varepsilon}(s)-R A(s) \bar{x}_{\varepsilon}(s)\right] d s \\
=\frac{1}{\varepsilon} \int_{-1}^{t_{-3 m / 2}}(E-R)\left(A(s) x_{\varepsilon}(s)\right) d s \\
+\frac{1}{\varepsilon} \int_{-1}^{t_{-3 m / 2}}(E-R)\left(\nu_{1}(s) \zeta_{1}(s, \varepsilon)\right) d s \\
\quad+\frac{1}{\varepsilon} \int_{-1}^{t_{-3 m / 2}}(E-R)\left(A(s)-\nu_{1}(s) I\right) \\
\quad \times\left(\varepsilon \eta_{1}(s, \varepsilon) \exp \left(\frac{1}{\varepsilon} \int_{-1}^{s} \nu_{1}(\tau, \varepsilon) d \tau\right)\right) \\
=J_{1}+J_{2}+J_{3} .
\end{aligned}
$$

To evaluate $J_{1}, J_{2}, J_{3}$, let us use the formula for the residual term in linear interpolation on $\left[t_{i}, t_{i+1}\right]$. According to this formula, for each function $f(s) \in$ $C^{2}[-1,1]$, for $s \in\left[t_{i}, t_{i+1}\right]$ we have

$$
[(E-R) f(s)]^{j}=\frac{\left[f^{\prime \prime}(\eta)\right]^{j}}{2}\left(s-t_{i}\right)\left(s-t_{i+1}\right), \quad \eta \in\left[t_{i}, t_{i+1}\right],
$$

where $j$ is a number of a component of the vector $f=\left(f^{1}, f^{2}, \ldots, f^{n}\right)$.

Due to (4.2) we have $\left|\bar{x}_{\varepsilon}(s)\right| \leq C$. By virtue of this fact and (4.17), (4.20), for $f=\bar{x}_{\varepsilon}$ we get

$$
J_{1} \leq C \varepsilon h^{2} .
$$

Relations (1.7) and (1.8) imply that the second derivative of the function located under the symbol $(E-R)$ in the expression for $J_{3}$ is estimated by $C / \varepsilon$. Considering this estimate and formulas (4.20) and (4.17), we obtain

$$
J_{3} \leq C \varepsilon h^{2} .
$$

Let us estimate $J_{2}$. Choose a number $j$ in such a way that $b_{1, j}(-1) \geq C>0$ holds for the $j$ th component. Due to the smoothness of $b_{1}(t)$, for any $\eta \in\left[-1, t_{-3 m / 2}\right]$ 
with $\varepsilon$ small we have $b_{1, j}(\eta, \varepsilon) \geq C>0$. Therefore from (1.6)-(1.8) and (4.9) we get

$$
-\frac{1}{2}\left[\nu_{1}(\eta) \zeta_{1}^{\prime \prime}(\eta, \varepsilon)\right]^{j} \geq \varepsilon^{-2} C \exp \left(\frac{1}{\varepsilon} \int_{-1}^{\eta} \nu_{1}(s) d s\right) \geq C \varepsilon^{-2}, \quad C>0,
$$

for $\eta \in\left[t_{i}, t_{i+1}\right](-2 m \leq i \leq-3 m / 2)$ (since by virtue of (4.17) $\eta+1 \leq C \varepsilon$ and $\left.\nu_{1}(\eta) \leq-\nu_{0}<0\right)$.

From (4.23) and (4.20), for $s \in\left[t_{i}, t_{i+1}\right](-2 m \leq i \leq-3 m / 2)$ we have

$$
\left[(E-R)\left(\nu_{1}(s) \zeta_{1}(s, \varepsilon)\right)\right]^{j} \geq C \varepsilon^{-2}\left(s-t_{i}\right)\left(t_{i+1}-s\right) .
$$

By (4.24) and (4.17) we obtain

$$
\begin{aligned}
& \frac{1}{\varepsilon} \int_{-1}^{t_{-3 m / 2}}\left[(E-R)\left(\nu_{1}(s) \zeta_{1}(s, \varepsilon)\right)\right]^{j} \\
& \quad \geq \frac{1}{\varepsilon^{3}} \sum_{i=-2 m}^{-3 m / 2-1} \int_{t_{i}}^{t_{i+1}} C\left(s-t_{i}\right)\left(t_{i+1}-s\right) d s \\
& \quad=\frac{C}{\varepsilon^{3}} \sum_{i=2 m}^{-3 m / 2-1} \frac{\left(t_{i+1}-t_{i}\right)^{3}}{6} \geq \frac{C h^{3} m}{2} \geq C h^{2}, \quad C>0 .
\end{aligned}
$$

However, (4.25) contradicts (4.13)-(4.19) and (4.21)-(4.22). Theorem 4 is proved.

\section{Preliminary Results}

This section is devoted to preparations for proving Theorem 5 .

\subsection{On series and finite sums estimates.}

Proposition 5.1. For every $\gamma$ and $\delta(1<\gamma \leq \delta)$ there is a constant $C(\gamma, \delta)$ such that, for all numbers $k$ and $s$,

$$
\sum_{i=-\infty}^{+\infty} \frac{1}{(1+|k-i|)^{\gamma}(1+|i-s|)^{\delta}} \leq \frac{C(\gamma, \delta)}{(1+|k-s|)^{\gamma}} .
$$

Proof. Let $k<s$. Let us divide the sum in the left side of the inequality into four sums, $\sum_{1}, \sum_{2}, \sum_{3}, \sum_{4}$, in accordance with the change in $i: i \in(-\infty, k]$ for $\sum_{1}$; $i \in(k, k+[(s-k) / 2])$ for $\sum_{2} ; i \in[k+[(s-k) / 2]+1, s]$ for $\sum_{3}$; and $i \in[s+1, \infty)$ for $\sum_{4}$. We then obtain

$$
\sum_{1} \leq \frac{1}{(1+s-k)^{\delta}} \sum_{i=-\infty}^{k} \frac{1}{(1+|k-i|)^{\gamma}} \leq \frac{C(\gamma)}{(1+s-k)^{\delta}} \leq \frac{C(\gamma)}{(1+s-k)^{\gamma}} .
$$

The terms $\sum_{2}, \sum_{3}, \sum_{4}$ are estimated in the same way. The proposition is proved.

Proposition 5.2. Let the function $F(x)=f(k-x) g(x-s)$ be monotone increasing (decreasing) and continuous on the interval $[q, p]$. Then

$$
\sum_{i=q}^{p-1} F(i) \leq \int_{q}^{p} F(x) d x \quad\left(\sum_{i=q+1}^{p} F(i) \leq \int_{q}^{p} F(x) d x\right) .
$$

This is a modification of the Cauchy-MacLaurin criterion. 


\subsection{Some properties of singular exponents on the mesh $\Delta$.}

Lemma 5.1. For each $\gamma \in(0,1]$ and $q=m+1, \ldots, j(j \geq m+1)$ the estimates

$$
\exp \left(\kappa \nu_{0}\left(t-t_{j}\right) / \varepsilon\right)=O^{*}\left((q-m)^{3 \kappa} /(j-m)^{3 \kappa}\right), \quad t \in\left[t_{q}, t_{q+1}\right],
$$

are valid.

Proof. Due to Lemma 2.1 we have

$$
t_{j}-t \geq t_{j}-t_{q+1}=\sum_{\nu=q+1}^{j-1} h_{\nu} \geq \frac{3 \varepsilon}{\nu_{0}} \sum_{\nu=q+1}^{j-1} \frac{1}{\nu-m+2}=\frac{3 \varepsilon}{\nu_{0}} \ln ((j-m) /(q-m))+O(1) .
$$

Substituting this in the exponential we obtain (5.1). This proves the lemma.

Lemma 5.2. Let functions $g_{1}(t, \varepsilon), g_{2}(t, \varepsilon) \in C^{3}[-1,1]$ be such that for some $\kappa \in$ $(0,1]$

$$
\begin{array}{ll}
\left|d^{i} g_{1}(t, \varepsilon) / d t^{i}\right| \leq C \varepsilon^{-i} \exp \left(\kappa \nu_{0}\left(t-t_{j}\right) / \varepsilon\right), & t \in\left[t_{m+1}, t_{j}\right], 0 \leq i \leq 3, \\
\left|d^{i} g_{2}(t, \varepsilon) / d t^{i}\right| \leq C \varepsilon^{-i} \exp \left(\kappa \nu_{0}\left(t_{j}-t\right) / \varepsilon\right), & t \in\left[t_{j}, 1\right], 0 \leq i \leq 3 .
\end{array}
$$

Then there exist functions $Z_{1}(t, \varepsilon), Z_{2}(t, \varepsilon) \in S(\Delta, 2,1)$, approximating $g_{1}$ and $g_{2}$, such that

$$
\begin{aligned}
& \left\|\frac{d^{i}\left(Z_{1}-g_{1}\right)}{d t^{i}}\right\|_{L_{\infty}\left[t_{q}, t_{q+1}\right]} \\
& \quad \leq \frac{C \varepsilon^{-i}}{(j-m)^{3 \kappa}(q-m)^{3-i-3 \kappa}} \quad(i=0,1 ; m+1 \leq q \leq j-1)
\end{aligned}
$$

and

$$
\left\|\frac{d^{i}\left(Z_{2}-g_{2}\right)}{d t^{i}}\right\|_{L_{\infty}\left[t_{q}, t_{q+1}\right]} \leq \frac{C \varepsilon^{-i}}{(q-m)^{3-i}} \quad(i=0,1 ; q \geq j) .
$$

Proof. By virtue of an approximation theorem of de Boor [21] there is a function $Z_{1}(t, \varepsilon)$ such that for $m+1 \leq q \leq j-1$ and $i=0,1$

$$
\begin{aligned}
\left\|\frac{d^{i}\left(Z_{1}-g_{i}\right)}{d t^{i}}\right\|_{L_{\infty}\left[t_{q}, t_{q+1}\right]} & \leq C h^{3-i}\left\|g_{1}^{\prime \prime \prime}\right\|_{L_{\infty}\left[t_{q-1}, t_{q+2}\right]} \\
& \leq C(\varepsilon /(q-m))^{3-i} \varepsilon^{-3} \exp \left(\nu_{0}\left(t_{q}-t_{j}\right) / \varepsilon\right) .
\end{aligned}
$$

But, according to Lemma 5.1, $\exp \left(\nu_{0}\left(t_{q}-t_{j}\right) / \varepsilon\right) \leq C(q-m)^{3 \kappa} /(j-m)^{3 \kappa}$, implying (5.2). The estimate (5.3) is obtained similarly. The lemma is proved.

5.3. Properties of $N$-bases in trial spaces. Let us study the functions $N_{i j}$ from Lemma 3.4. Let $b_{p}(t)$ be an eigenvector of $A(t)$ and let

$$
N_{i j}=\sum_{p=1}^{n} \gamma_{p}^{i, j}(t) b_{p}(t)
$$

Lemma 5.3. The representations

$$
\gamma_{p}^{i, j}(t)=\delta_{j p} B_{i+1,1}(t)+\widehat{\Phi}_{i j}(t, \varepsilon)+\hat{\mu}_{i j}(t, \varepsilon)
$$

are valid, where $\delta_{q p}$ is the Kronecker symbol, $j, p=1, \ldots, n, t \in\left[t_{m+i_{0}+2}, 1\right] \cup$ $\left[-1, t_{-m-i_{0}-2}\right]$, and $i$ changes on the index set from Lemma 3.4. In addition, $\widehat{\Phi}_{i j}$ and $\hat{\mu}_{i j}$ satisfy formulas (3.5)-(3.8). 
Proof. Let the vectors $b_{p}^{*}(t)$ be such that $\left(b_{q}(t), b_{p}^{*}(t)\right)=\delta_{q p}$ for each fixed $t$ (here the inner product is considered in $R^{n}$ ). From (5.4), by simple computations, one can show that $\gamma^{i, j}(t)=\left(N_{i j}(t), b_{p}^{*}(t)\right)$. Hence by virtue of continuity and smoothness of $b_{p}(t)$ and by formulas (3.4)-(3.8), we get the lemma.

Denote the length of the $B$-spline support in the representation (3.4), (3.14), (3.15) of the function $N_{i j}$ by $Z_{i j}$. Let $L_{\varepsilon}^{*}=-\varepsilon d / d t-A^{T}(t)$ be the formal conjugate operator of $L_{\varepsilon}$.

Lemma 5.4. There exists a constant $C$ such that for any $x(t) \in\left(L_{\infty}[-1,1]\right)^{n}$, $\|x\|_{\infty} \leq 1$

$$
\left|\left(x, L_{\varepsilon}^{*} N_{i j}\right)\right| \leq C \max \left\{L_{i j}, \varepsilon\right\} .
$$

Proof. Let $L_{i j} \leq C \varepsilon$. Consider the case in which $N_{i j}$ has the representation (3.4) (the representations (3.14) and (3.15) are considered similarly). We have

$$
\left|\left(x, L_{\varepsilon}^{*} N_{i j}\right)\right| \leq\left\|L_{\varepsilon}^{*} N_{i j}\right\|_{1} \leq\left\|L_{\varepsilon}^{*} B_{i+1,1} b_{j}\left(t_{i+1}\right)\right\|_{1}+\left\|L_{\varepsilon}^{*} \Phi_{i j}(t)\right\|_{1}\left\|L_{\varepsilon}^{*} \mu_{i j}(t, \varepsilon)\right\|_{1} .
$$

Further, by virtue of (3.7) and the inequalities $\left\|B_{i+1,1}\right\|_{1} \leq C L_{i j}$ and $\left\|B_{i+1,1}^{\prime}\right\|_{1} \leq$ $C$, we have

$$
\left\|L_{\varepsilon}^{*} B_{i+1,1} b_{j}\left(t_{i+1}\right)\right\|_{1}+\left\|L_{\varepsilon}^{*} \Phi_{i j}\right\|_{1} \leq C\left(\varepsilon+L_{i j}\right)
$$

and, due to (3.8),

$$
\begin{aligned}
\left\|L_{\varepsilon}^{*} \mu_{i j}\right\|_{1} \leq & \sum_{|p| \geq m+1} \int_{t_{p}}^{t_{p+1}}\left\|\varepsilon \mu_{i j}^{\prime}-A(t) \mu_{i j}\right\|_{R^{n}} d t \\
\leq & \sum_{|p| \geq m+1} \frac{C_{\varepsilon}}{\max \left\{(|p|-m)^{3},(|i|-m)^{3}\right\}} \\
& \quad+\sum_{|p| \geq m+1} \frac{C h_{p}}{\max \left\{(|p|-m)^{3},(|i|-m)^{3}\right\}} .
\end{aligned}
$$

From (5.7) and (5.8) the estimate (5.6) follows for $L_{i j} \leq C \varepsilon$. In the case $L_{i j} \gg \varepsilon$, the representation (3.14) or (3.15) holds for $N_{i j}$, the term $\delta_{i j}$ is missing, and the term containing the $B$-spline is estimated similarly to (5.7). This proves the lemma.

Lemma 5.5. For any $p$ and $q$ with $m+i_{0} \leq p \leq q \leq 2 m-2$, and for $j=1,2, \ldots, n$, the estimates

$$
\left\|L_{\varepsilon}^{*} \sum_{\nu=p}^{q} N_{\nu j}\right\|_{1} \leq C \varepsilon \ln ((q-m) /(p-m))
$$

are valid.

Proof. We have

$$
L_{\varepsilon}^{*} \sum_{\nu=p}^{q} N_{\nu j}(t)=L_{\varepsilon}^{*} \sum_{\nu=p}^{q} B_{\nu+1,1}(t) e_{j}\left(t_{\nu+1}\right)+L_{\varepsilon}^{*} \sum_{\nu=p}^{q} \Phi_{\nu j}(t)+L_{\varepsilon}^{*} \sum_{\nu=p}^{q} \mu_{\nu j}(t) .
$$

Further, by virtue of Lemmas 3.4, 2.1 and the above relation, we have

$$
\sum_{\nu=p}^{q} B_{\nu+1,1}(t) \equiv 1 \quad \text { for } t \in\left[t_{p+2}, t_{q+1}\right],
$$


and

$$
\begin{aligned}
& \left\|L_{\varepsilon}^{*} \sum_{\nu=p}^{q} B_{\nu+1,1}(t) e_{j}\left(t_{\nu+1}\right)\right\|_{1} \leq \varepsilon\left\|B_{p+1,1}^{\prime}(t) e_{j}\left(t_{p+1}\right)\right\|_{1} \\
& \quad \leq C\left(\varepsilon+t_{q+2}-t_{p+1}\right) \leq C \varepsilon \ln ((q-m) /(p-m)) .
\end{aligned}
$$

Next, according to (3.6), (3.7) and Lemma 2.1,

$$
\begin{aligned}
\| L_{\varepsilon}^{*} \sum_{\nu=p}^{q} \Phi_{\nu j}(t, \varepsilon) & \\
& \leq \varepsilon\left\|\sum_{\nu=p}^{q} \Phi_{\nu j}^{\prime}(t, \varepsilon)\right\|_{1}+C\left\|\sum_{\nu=p}^{q} \Phi_{\nu j}(t, \varepsilon)\right\|_{1} \\
& \leq \varepsilon \sum_{\kappa=p+1}^{q+2} \int_{t_{\kappa}}^{t_{\kappa+1}} \sum_{\nu=p}^{q}\left\|\Phi_{\nu j}^{\prime}\right\|_{R^{n}} d t+C \sum_{\kappa=p+1}^{q+2} \int_{t_{\kappa}}^{t_{\kappa+1}}\left\|\Phi_{\nu j}\right\|_{R^{n}} d t \\
& \leq C \varepsilon \sum_{\kappa=p+1}^{q+2} 1 /(\kappa-m)+C\left(t_{q+3}-t_{p+1}\right) \\
& \leq C \varepsilon \ln ((q-m) /(p-m)) .
\end{aligned}
$$

Finally,

$$
\begin{aligned}
\| L_{\varepsilon}^{*} & \sum_{\nu=p}^{q} \mu_{\nu j}(t, \varepsilon) \|_{1} \\
& \leq \varepsilon \sum_{\kappa=m+1}^{2 m-1} \int_{t_{\kappa}}^{t_{\kappa+1}} \sum_{\nu=p}^{q}\left\|\mu_{\nu j}^{\prime}(t, \varepsilon)\right\|_{R^{n}} d t+C \sum_{\kappa=m+1}^{2 m-1} \int_{t_{\kappa}}^{t_{\nu+1}} \sum_{\nu=p}^{q}\left\|\mu_{\nu j}(t, \varepsilon)\right\|_{R^{n}} d t \\
& \leq \sum_{\kappa=m+1}^{2 m-1} \sum_{\nu=p}^{q} \frac{C \varepsilon}{\max \left\{(\kappa-m)^{3},(\nu-m)^{3}\right\}} \\
& \leq \sum_{\kappa=m+1}^{2 m-1} \sum_{\nu=m}^{2 m-1} \frac{C \varepsilon}{\max \left\{(\kappa-m)^{3},(\nu-m)^{3}\right\}} \\
& \leq C \varepsilon \sum_{\kappa=m+1}^{2 m-1} \frac{1}{(\kappa-m)^{3}} \leq C \varepsilon .
\end{aligned}
$$

The lemma is proved.

\subsection{Properties of bases which are biorthogonal to bases $\left\{N_{i j}\right\}$.}

Lemma 5.6. In the space $F$ there are bases $\left\{\lambda_{i j}(t)\right\}$ which are biorthogonal to the bases $\left\{N_{i j}\right\}$ (i.e., $\left.\left(N_{i j}, \lambda_{p s}\right)=\delta_{i j} \delta_{p s}\right)$; moreover,

$$
\lambda_{i j}(t)=\sum_{s=1}^{n} \sum_{p=-2 m-2}^{2 m-2} \gamma_{p s}^{i j} N_{p s},
$$


where

$$
\begin{aligned}
& \left|\gamma_{p s}^{i j}\right| \leq C /\left((1+|i-p|)^{2} \max \left\{L_{i j}, L_{p s}\right\}\right), \\
& \left|\gamma_{p s}^{i j}\right| \leq C /\left((1+|i-p|)^{5 / 2}\left(L_{i j} L_{p s}\right)^{1 / 2}\right) .
\end{aligned}
$$

This lemma was proved in [11].

Lemma 5.7. There exists a constant $C$ such that for every $i, j$ and $\nu$ with $-2 m-2 \leq i \leq 2 m-2,1 \leq j<n,-2 m \leq \nu \leq 2 m-1$, and for every $t \in$ $\left[t_{\nu}, t_{\nu+1}\right]$, the following estimates hold:

$$
\begin{aligned}
& \left\|\lambda_{i j}(t)\right\|_{R^{n}} \leq C /\left(L_{i j}(1+|i-\nu|)^{2}\right), \\
& \left\|\lambda_{i j}(t)\right\|_{R^{n}} \leq C /\left(\left(L_{i j} \max _{1 \leq s \leq n} L_{\nu s}\right)^{1 / 2}(1+|i-\nu|)^{5 / 2}\right) .
\end{aligned}
$$

Proof. Applying Lemma 5.6, we have

$$
\begin{aligned}
\left\|\lambda_{i j}(t)\right\|_{R^{n}} & \leq \sum_{s=1}^{n} \sum_{p=-2 m-2}^{2 m-2}\left|\gamma_{p s}^{i j}\right|\left\|N_{p s}(t)\right\|_{R^{n}} \\
& \leq C \sum_{s=1}^{n} \sum_{p=\nu-2}^{\nu}\left|\gamma_{p s}^{i j}\right|\left\{\left|B_{p}(t)\right|+\left\|\mu_{p s}(t)\right\|_{R^{n}}\right\}
\end{aligned}
$$

where $B_{p}(t)$ denotes a $B$-spline of the first or second degree in the corresponding basis function representation.

Further, due to (5.10), Lemma 3.4 and Proposition 5.1,

$$
\begin{aligned}
& \sum_{s=1}^{n} \sum_{p=-2 m-2}^{2 m-2}\left|\gamma_{p s}^{i j}\right|\left\|\mu_{p s}(t)\right\|_{R^{n}}=\sum_{s=1}^{n} \sum_{m+i_{0} \leq|p| \leq 2 m}\left|\gamma_{p s}^{i j}\right|\left\|\mu_{p s}(t)\right\|_{R^{n}} \\
& \quad \leq C / L_{i j} \sum_{s=1}^{n} \sum_{m+i_{0} \leq|p| \leq 2 m} 1 /\left((1+|p-i|)^{2} \max \left\{(|p|-m)^{3},(|| \nu|-m|+1)^{3}\right\}\right) \\
& \quad \leq C /\left(L_{i j} \max \left\{(1+|i-m|)^{2},(1+|| \nu|-m|)^{3}\right\}\right) \leq C /\left(L_{i j}(1+|i-\nu|)^{2} .\right.
\end{aligned}
$$

The similar estimate of the first term in (5.14) follows from (5.10). Using a similar argument, the estimate (5.12) can be proved.

Using (5.1), we can prove (5.13) in the same way. The proof is completed.

\section{Lemma 5.8.}

$$
\left\|\lambda_{i j}\right\|_{1} \leq C .
$$

Proof. Lemma 3.4 implies that $\left\|N_{p s}\right\|_{1} \leq C L_{p s}$. Hence by (5.10) we have

$$
\begin{aligned}
\left\|\lambda_{i j}\right\|_{1} & \leq \sum_{s=1}^{n} \sum_{p=-2 m-2}^{2 m-2}\left|\gamma_{p s}^{i j}\right|\left\|N_{p s}\right\|_{1} \\
& \leq C \sum_{s=1}^{n} \sum_{p=-2 m-2}^{2 m-2} L_{p s} /\left((1+|i-p|)^{2} \max \left\{L_{p s}, L_{i j}\right\}\right) \\
& \leq C \sum_{s=1}^{n} \sum_{p=-2 m-2}^{2 m-2} 1 /(1+|i-p|)^{2} \leq C .
\end{aligned}
$$

The lemma is proved. 
Lemma 5.9.

$$
\left\|G_{\varepsilon} \lambda_{i j}\right\|_{\infty} \leq C \min \left\{1 / \varepsilon, 1 / L_{i j}\right\} .
$$

Proof. From the inequalities $\left\|G_{\varepsilon}(t, \xi)\right\|_{\infty} \leq C \varepsilon$ and $\left\|N_{p s} / L_{p s}\right\|_{1} \leq C$ and Lemma 5.6 we get

$$
\begin{aligned}
\left\|G_{\varepsilon} \lambda_{i j}(t)\right\|_{R^{n}} & \leq \sum_{s=1}^{n} \sum_{p=-2 m-2}^{2 m-2}\left|\gamma_{p s}^{i j}\right|\left\|G_{\varepsilon} N_{p s}(t)\right\|_{R^{n}} \\
& \leq C \sum_{s=1}^{n} \sum_{p=-2 m-2}^{2 m-2}\left\|G_{\varepsilon}\left(N_{p s} / L_{p s}\right)\right\|_{R^{n}} /(1+|i-p|)^{2} \\
& \leq C / \varepsilon \sum_{s=1}^{n} \sum_{p=-2 m-2}^{2 m-2}\left\|N_{p s} / L_{p s}\right\|_{1} /(1+|i-p|)^{2} \leq C \varepsilon .
\end{aligned}
$$

By virtue of Lemma 1.1, $\left\|G_{\varepsilon}\right\|_{L_{\infty} \rightarrow L_{\infty}} \leq C$, and hence

$$
\left\|G_{\varepsilon} \lambda_{i j}(t)\right\|_{\infty} \leq C\left\|\lambda_{i j}\right\|_{\infty} \leq C / L_{i j} .
$$

The lemma follows from the last inequalities. The proof is completed.

\subsection{Some properties of the Green function in the problem (1.1)-(1.2).}

5.5.1. Expansion of the Green function in the eigenvectors of the matrix $A(t)$. Let $b_{\nu}(t)$ be the eigenvectors of $A(t)$, and expand $G_{\varepsilon}(t, \xi)$ in the following way:

$$
G_{\varepsilon}(t, \xi)=\sum_{\nu=1}^{n} \beta_{\nu}(t, \xi) e_{\nu}^{T}(\xi) .
$$

Lemma 5.10. The estimates $(\nu=1,2, \ldots, n)$

$$
\left\|\frac{\partial^{q}}{\partial \xi^{q}} \beta_{\nu}(t, \xi)\right\|_{R^{n}} \leq \frac{C}{\varepsilon^{1+q}} \exp \left(-\nu_{0}|t-\xi| / \varepsilon\right), \quad q=0,1
$$

hold.

Proof. This follows from Lemma 1.1, the relation $\beta_{\nu}(t, \xi)=\left(G_{\varepsilon}(t, \xi), b_{\nu}^{*}(\xi)\right)$ (see the proof of Lemma 5.3) and the smoothness of the vector $b_{\nu}^{*}(\xi)$.

5.5.2. On spline approximation of the Green function. Let $t \in\left[t_{s}, t_{s+1}\right] \subset\left[t_{m+i_{0}+2}, 1\right]$. We shall construct two specific spline approximations of the function $G_{\varepsilon}(t, \xi)$ as a function of $\xi$ when $t$ is fixed.

Lemma 5.11. There is a matrix function $Z_{1}(t)$ such that:

1. For each fixed $t$, the rows of $Z_{1}(t)$ are elements of the space $F$.

2. $\max _{\xi \in\left[t_{\nu}, t_{\nu+1}\right]}\left\|Z_{1}(t, \xi)-G_{\varepsilon}(t, \xi)\right\|_{R^{n \times n}} \leq C /\left(\varepsilon(\nu-m)^{2}\right), \quad s \leq \nu \leq 2 m-1$.

3. $\operatorname{supp} Z_{1}(t, \xi) \subset\left[t_{*}, 1\right]$, where $t_{*}=t_{s-[(s-m-1) / 2]}(t$ is fixed, $\xi$ changes $)$.

4. $\left\|Z_{1}(t, \xi)\right\|_{L_{\infty}} \leq C / \varepsilon(-1 \leq t, \xi \leq 1)$.

Lemma 5.12. There is a matrix function $Z_{2}(t, \xi)$ such that:

1. The rows of $Z_{2}(t, s)$ are elements of the space $F$ for every fixed $t$.

2. $\max _{t \in\left[t_{\nu}, t_{\nu+1}\right]}\left\|Z_{2}(t, \xi)-G_{\varepsilon}(t, \xi)\right\|_{R^{n \times n}} \leq C /\left(\varepsilon(s-m)^{2}\right), m+1 \leq \nu \leq s$.

3. $\operatorname{supp} Z_{2}(t, \xi) \subset\left[t_{m+1}, t_{*}\right]$, where $t_{*} \geq t_{s} ; t_{s}$ is a knot such that either $t_{*}=1$ or $t_{*}-t_{s}=O^{*}(\varepsilon)$.

4. $\left\|Z_{2}(t, \xi)\right\|_{L_{\infty}} \leq C / \varepsilon(-1 \leq t, \xi \leq 1)$. 
Let us prove Lemma 5.11. The proof of Lemma 5.12 is similar.

Consider an arbitrary row $g_{\varepsilon}(t, \xi)$ of the matrix $G_{\varepsilon}(t, \xi)$ for some fixed $t$. For this row let us consider the problem $L_{\varepsilon} \delta(\xi)=\left[g_{\varepsilon}(t, \xi)\right]^{T}$ with boundary conditions (1.2). The solution $\delta_{\varepsilon}(t, \xi)$ of this problem may be estimated as follows:

$$
\begin{aligned}
\left\|\delta_{\varepsilon}(t, \xi)\right\|_{R^{n}}=\left\|\int_{-1}^{1} G_{\varepsilon}(\xi, \tau) g_{\varepsilon}(t, \tau) d \tau\right\|_{R^{n}} \\
\leq \\
\leq \frac{C}{\varepsilon^{2}} \int_{-1}^{1} \exp \left(-\nu_{0}|\xi-\tau| / \varepsilon\right) \exp \left(-\nu_{0}|t-\tau| / \varepsilon\right) d \tau \\
\leq \frac{C}{\varepsilon}\left(1+\frac{|t-\tau|}{\varepsilon} \exp \left(-\nu_{0} \frac{|t-\tau|}{\varepsilon}\right)\right) \\
\quad \leq C(\kappa) / \varepsilon \exp \left(-\kappa \nu_{0}|t-\xi| / \varepsilon\right)
\end{aligned}
$$

for every $\kappa \in(0,1)$. Analogous estimates are also valid for $\partial^{j} \delta_{\varepsilon}(t, \xi) / \partial \xi^{j}, j=1,2,3$.

Letting $\kappa=2 / 3$, from Lemma 5.1 we get that there is a spline $\delta_{\varepsilon, m}(t, \xi)$ defined as a function of $\xi$ on $\left(t_{s+1}, 1\right]$ and satisfying (for $\left.i=0,1\right)$ the inequality

$$
\left\|\left(\delta_{\varepsilon, m}(t, \xi)-\delta_{\varepsilon}(t, \xi)\right)^{(i)}\right\|_{L_{\infty}\left[t_{\nu}, t_{\nu+1}\right]} \leq \frac{C}{\varepsilon^{i+1}(\nu-m)^{2}}, \quad \nu \geq s .
$$

Continue the functions $\delta_{\varepsilon, m}$ on the interval $[-1,1]$ in such a way that the quantity $\varepsilon\left\|\dot{\delta}_{\varepsilon, m}\right\|_{\infty}+\left\|\delta_{\varepsilon, m}\right\|_{\infty}$ is controlled. With this aim in mind, take a point $t_{*}=t_{\mu}$ $(\mu=s-[(s-m-1) / 2])$. It is easy to show that $t_{s}-t_{*}=O^{*}(\varepsilon)$. Let $t_{* *}$ be a closer knot to the middle of interval $\left[t_{*}, t_{s}\right]$. Then $t_{* *}-t_{*}=O^{*}(\varepsilon)$ and $t_{s}-t_{* *}=O^{*}(\varepsilon)$. By using $t_{*}, t_{* *}$, and $t_{s}$ we construct "patch-functions" (see [7]) $\widetilde{Z}_{1}(t)$ and $\widetilde{Z}_{2}(t)$, parabolic splines and for which

$$
\begin{aligned}
\widetilde{Z}_{j}^{(i)}\left(t_{*}\right)=0 & (i, j=0,1), \\
\widetilde{Z}_{1}\left(t_{s}\right) & =0, \quad \widetilde{Z}_{1}^{\prime}\left(t_{s}\right)=1, \quad \widetilde{Z}_{2}\left(t_{s}\right)=1, \quad \widetilde{Z}_{2}^{\prime}\left(t_{s}\right)=0 .
\end{aligned}
$$

From estimates obtained in [7] it follows that

$$
\left\|\widetilde{Z}_{1}^{(i)}(t)\right\|_{\infty} \leq C \varepsilon^{1-i}, \quad\left\|\widetilde{Z}_{2}^{(i)}(t)\right\|_{\infty} \leq C \varepsilon^{-i} \quad(i=0,1) .
$$

For $t \in\left[t_{*}, t_{s}\right]$ we put

$$
\delta_{\varepsilon, m}(t, \xi)=\delta_{\varepsilon, m}\left(t, t_{s+0}\right) \widetilde{Z}_{2}(\xi)+\delta_{\varepsilon, m}^{\prime}\left(t, t_{s+0}\right) Z_{1}(\xi)
$$

The continued function is sewn smoothly into the point $t_{s}$ and vanishes with its derivative at the point $t_{*}$. Obviously it is possible to consider this function as defined in the whole interval $[-1,1]$, if we put $\delta_{\varepsilon, m}(t)=0$ for $t \in\left[-1, t_{\mu}\right]$. From estimates of $g_{\varepsilon}(t, \xi)$ (see Lemma 1.1) and the definition of $\delta_{\varepsilon, m}(t, \xi)$ it follows that

$$
\left\|\delta_{\varepsilon, m}^{(i)}\left(t, t_{s+0}\right)\right\|_{R^{n}} \leq C / \varepsilon^{1+i}
$$

From (5.20)-(5.22) we obtain

$$
\left\|L_{\varepsilon} \delta_{\varepsilon, m}(t, \xi)\right\|_{L_{\infty}\left[t_{*}, t_{s}\right]} \leq C \varepsilon
$$

Now let $Z_{1}(t, \xi)$ be the matrix whose rows are the vectors $L_{\varepsilon} \delta_{\varepsilon, m}(t, \xi)$. Then from (5.19) and (5.13) we let Lemma 5.11.

Let us establish two estimates on the approximation of the functions $G_{\varepsilon}(t, \xi)$ and $Z_{p}(t, \xi)$. 
Lemma 5.13. The following estimates hold:

$$
\begin{aligned}
& \left\|\int_{-1}^{1}\left(G_{\varepsilon}(t, \xi)-Z_{1}(t, \xi)\right) \lambda_{i j}(\xi) d \xi\right\|_{R^{n}} \\
& \quad \leq \frac{C}{\varepsilon}\left(\frac{1}{(i-m)^{3 / 2}}+\frac{1}{(1+|i-s|)^{3 / 2}}\right)
\end{aligned}
$$

for $t \in\left[t_{s}, t_{s+1}\right], m+i_{0}+2 \leq s \leq i+2$; and

$$
\begin{aligned}
& \left\|\int_{-1}^{1}\left(G_{\varepsilon}(t, \xi)-Z_{2}(t, \xi)\right) \lambda_{i j}(\xi) d \xi\right\|_{R^{n}} \\
& \quad \leq \frac{C}{\varepsilon}\left(\frac{1}{(i-m)^{3 / 2}}+\frac{1}{(1+|i-s|)^{3 / 2}}\right)
\end{aligned}
$$

for $t \in\left[t_{s}, t_{s+1}\right], m+i_{0}+2 \leq i+2 \leq s \leq 2 m-1$.

Proof. Let us prove (5.24). The estimate (5.25) is established in a similar way. We have

$$
\begin{aligned}
& \left\|\int_{-1}^{1}\left(G_{\varepsilon}(t, \xi)-Z_{1}(t, \xi)\right) \lambda_{i j}(\xi) d \xi\right\|_{R^{n}} \\
& \leq \int_{-1}^{t}\left(G_{\varepsilon}(t, \xi)-Z_{1}(t, \xi)\left\|_{R^{n \times n}}\right\| \lambda_{i j}(\xi) \|_{R^{n}} d \xi\right. \\
& \quad+\int_{t}^{1}\left\|G_{\varepsilon}(t, \xi)-Z_{1}(t, \xi)\right\|_{R^{n \times n}}\left\|\lambda_{i j}(\xi)\right\|_{R^{n}} d \xi .
\end{aligned}
$$

Further, due to (5.13),

$$
\begin{gathered}
\int_{-1}^{t}\left\|G_{\varepsilon}(t, \xi)-Z_{1}(t, \xi)\right\|_{R^{n \times n}}\left\|\lambda_{i j}(\xi)\right\|_{R^{n}} d \xi \\
\leq \int_{-1}^{t}\left\|G_{\varepsilon}(t, \xi)\right\|_{R^{n \times n}}\left\|\lambda_{i j}(\xi)\right\| d \xi+\int_{-1}^{t}\left\|Z_{1}(t, \xi)\right\|_{R^{n \times n}}\left\|\lambda_{i j}(\xi)\right\|_{R^{n}} d \xi \\
\int_{-1}^{t}\left\|G_{\varepsilon}(t, \xi)\right\|_{R^{n \times n}}\left\|\lambda_{i j}(\xi)\right\|_{R^{n}} d \xi \\
\leq \frac{C}{\varepsilon} \int_{-1}^{t} \exp \left(-\nu_{0}|\xi-t| / \varepsilon\right)\left\|\lambda_{i j}(\xi)\right\|_{R^{n}} d \xi \\
=\frac{C}{\varepsilon}\left(\int_{-1}^{t_{m}}+\int_{t_{m}}^{t_{m+1}}+\int_{t_{m+1}}^{t}\right) .
\end{gathered}
$$

Since $\varepsilon|\ln \varepsilon| \ll 1 / m$ and $t \in\left[t_{m+1}, 1\right]$, we have $\exp \left(-\nu_{0}|\xi-t| / \varepsilon\right) \leq C \varepsilon^{2}$ for $\xi \in\left[-1, t_{m}\right]$. Thus, according to (5.15),

$$
1 / \varepsilon \int_{-1}^{t_{m}} \exp \left(-\nu_{0}|t-\xi|\right)\left\|\lambda_{i j}(\xi)\right\|_{R^{n}} d \xi \leq C \varepsilon .
$$

Further, due to (5.13),

$$
\begin{aligned}
& \frac{1}{\varepsilon} \int_{t_{m}}^{t_{m+1}} \exp \left(-\nu_{0}|\xi-t| / \varepsilon\right)\left\|\lambda_{i j}(\xi)\right\|_{R^{n}} d \xi \leq C\left\|\lambda_{i j}(\xi)\right\|_{L_{\infty}\left[t_{m}, t_{m+1}\right]} \\
& \quad \leq \frac{C(i-m)^{1 / 2}}{\varepsilon|\ln \varepsilon|(i-m)^{5 / 2}} \leq \frac{C}{\left(\varepsilon(i-m)^{2}\right)}
\end{aligned}
$$


Now applying Lemmas 5.1, 5.7, 2.1 and Proposition 5.2 we obtain

$$
\begin{aligned}
& \frac{1}{\varepsilon} \int_{t_{m+1}}^{t} \exp \left(-\nu_{0}|\xi-t| / \varepsilon\right)\left\|\lambda_{i j}(\xi)\right\|_{R^{n}} d \xi \\
& \leq \frac{C}{\varepsilon} \sum_{p=m+1}^{s} \int_{t_{p}}^{t_{p+1}} \exp \left(-\nu_{0}|\xi-t| / \varepsilon\right)\left\|\lambda_{i j}(\xi)\right\|_{R^{n}} d \xi \\
& \leq \frac{C}{\varepsilon} \sum_{p=m+1}^{s} \frac{h_{p}(p-m)^{3}(p-m)^{1 / 2}(i-m)^{1 / 2}}{\varepsilon(s-m)^{3}(1+|i-p|)^{5 / 2}} \\
& \leq \frac{C}{\varepsilon} \sum_{p=m+1}^{s} \frac{\varepsilon(p-m)^{3}(p-m)^{1 / 2}(i-m)^{1 / 2}}{(p-m) \varepsilon(s-m)^{3}(1+|i-p|)^{5 / 2}} \\
& \leq \frac{C}{\varepsilon} \frac{(i-m)^{1 / 2}}{(s-m)^{1 / 2}} \sum_{p=m+1}^{s} \frac{1}{(1+i-p)^{5 / 2}} \\
& \quad \leq \frac{C}{\varepsilon}\left\{\begin{array}{l}
(s-m) /(1+i-s)^{5 / 2}, \\
1 /(1+i-s)^{3 / 2}, \quad s-m>i-s,
\end{array}, \quad s,\right. \\
& \leq C /\left(\varepsilon(1+i-s)^{3 / 2}\right) .
\end{aligned}
$$

By means of property 4 from Lemma 5.11 and (5.13) we get analogously

$$
\begin{aligned}
\int_{-1}^{t} & \left\|Z_{1}(t, \xi)\right\|_{R^{n \times n}}\left\|\lambda_{i j}(\xi)\right\|_{R^{n}} d \xi=\int_{t_{*}}^{t}\left\|Z_{1}(t, \xi)\right\|_{R^{n \times n}}\left\|\lambda_{i j}(\xi)\right\|_{R^{n}} d \xi \\
\leq & \frac{C}{\varepsilon} \int_{t_{*}}^{t}\left\|\lambda_{i j}(\xi)\right\|_{R^{n}} d \xi \\
\leq & \frac{C}{\varepsilon} \sum_{p=s^{\prime}}^{s} \int_{t_{p}}^{t_{p+1}}((p-m)(i-m))^{1 / 2} /(1+|i-p|)^{5 / 2} \varepsilon^{-1} d \xi \\
\leq & \frac{C}{\varepsilon}(i-m)^{1 / 2} \sum_{p=s^{\prime}}^{s} \frac{1}{(1+i-p)^{5 / 2}(p-m)^{1 / 2}} \\
& \leq \frac{C}{\varepsilon} \frac{(i-m)^{1 / 2}}{(s-m)^{1 / 2}} \sum_{p=s^{\prime}}^{s} \frac{1}{(1+i-p)^{5 / 2}} \leq \frac{C}{\varepsilon(1+i-s)^{3 / 2}},
\end{aligned}
$$

where $s^{\prime}=s-[(s-m-1) / 2]$.

From (5.27) and (5.32) we obtain the estimate

$$
\begin{array}{r}
\int_{-1}^{t}\left\|G_{\varepsilon}(t, \xi)-Z_{1}(t, \xi)\right\|_{R^{n \times n}}\left\|\lambda_{i j}(\xi)\right\|_{R^{n}} d \xi \\
\leq \frac{C}{\varepsilon}\left(1 /(1+i-s)^{3 / 2}+1 /(i-m)^{3 / 2}\right) .
\end{array}
$$


Due to property 2 from Lemma 5.11, estimate (5.11), Lemma 2.1 and Proposition 5.1, we have

$$
\begin{aligned}
\int_{t}^{1} \| & G_{\varepsilon}(t, \xi)-Z_{1}(t, \xi)\left\|_{R^{n \times n}}\right\| \lambda_{i j}(\xi) \|_{R^{n}} d \xi \\
& \leq \frac{C}{\varepsilon} \sum_{\nu=s}^{2 m-1} \frac{1}{(\nu-m)^{3}} \frac{\varepsilon}{\nu-m} \frac{(i-m)^{1 / 2}(\nu-m)^{1 / 2}}{\varepsilon /(1+|i-\nu|)^{5 / 2}} \\
& =\frac{C(i-m)^{1 / 2}}{\varepsilon} \sum_{\nu=s}^{2 m-1} \frac{1}{(\nu-m)^{5 / 2}}(1+|i-\nu|)^{5 / 2} \\
& \leq \frac{C}{\varepsilon(i-m)^{2}} .
\end{aligned}
$$

From (5.33) and (5.34) we obtain (5.24). The lemma is proved.

5.5.3. On decomposition of the matrix $Z_{p}(t, \xi)$ in the basis functions of the space $F$. Since each row $Z_{p}(t, \xi)(p=1,2)$ is an element of $F$, it may be decomposed in the basis $\left\{N_{i j}^{T}\right\}$, i.e.,

$$
Z_{p}(t, \xi)=\sum_{j=1}^{n} \sum_{i=-2 m-2}^{2 m-2} \alpha_{i j}^{p}(t) N_{i j}^{T}(\xi),
$$

where the $\alpha_{i j}^{p}(t)$ are column vectors. Let us study the coefficients $\alpha_{i j}^{p}(t)$.

Lemma 5.14. Let $t \in\left[t_{s}, t_{s+1}\right] \subset\left[t_{m+i_{0}+2}, 1\right]$. Then

$$
\alpha_{i j}^{p}(t)=\beta_{j}\left(t, t_{i+2}\right)\left(1+O\left(\frac{1}{|i|-m}\right)+O\left(\frac{1}{\varepsilon(|i|-m)^{2}}\right)\right)
$$

(if $s \leq i+2$, then for $p=1$; if $s>i+2$, then for $p=2$ ), where $\beta_{j}(t, \xi)$ is the function from (5.17).

Proof. To be specific, let $s \leq i+2$. For $\xi \in\left[t_{m+i_{0}+2}, 1\right]$ we have

$$
Z_{1}(t, \xi)=\sum_{j=1}^{n} \sum_{\kappa=m+i_{0}}^{2 m-2} \alpha_{\kappa j}^{1}(t) N_{\kappa j}^{T}(\xi),
$$

since $N_{\kappa j}(\xi)=0$ for $\kappa<m+i_{0}$ and $\xi \in\left[t_{m+i_{0}+2}, 1\right]$ (see Lemma 3.4).

Further, in accordance with Lemma 5.11,

$$
Z_{1}(t, \xi)=G_{\varepsilon}(t, \xi)+O\left(1 /\left(\varepsilon(i-m)^{2}\right), \quad \xi \in\left[t_{\nu}, t_{\nu+1}\right], \nu \geq s+1 .\right.
$$

Substitute $\xi=t_{\nu}$ in this representation and apply (5.37). Then

$$
\sum_{j=1}^{n} \sum_{\kappa=m+i_{0}}^{2 m-2} \alpha_{\kappa j}^{1}(t) N_{\kappa j}^{T}(\xi)=G_{\varepsilon}\left(t, t_{\nu}\right)+O\left(1 /\left(\varepsilon(\nu-m)^{2}\right)\right.
$$

for $\nu=s+1, s+2, \ldots, 2 m$. To simplify (5.39) we use the decompositions obtained in Lemmas 5.3 and 5.10, and write

$$
N_{\kappa j}^{T}(t)=\sum_{q=1}^{n} \gamma_{q}^{\kappa, j}(t) e_{q}(t), \quad G_{\varepsilon}\left(t, t_{\nu}\right)=\sum_{p=1}^{n} \beta_{q}\left(t, t_{\nu}\right) e_{q}\left(t_{\nu}\right) .
$$


Substitute these decompositions in (5.39) and equate the coefficients of the vectors $e_{q}\left(t_{\nu}\right)$. In accordance with (5.5) we get

$$
\begin{array}{r}
\sum_{\kappa=m+i_{0}}^{2 m-2}\left(\delta_{\nu \kappa+2}+\widehat{\Phi}_{\kappa j}\left(t_{\nu}\right)+\mu_{\kappa j}\left(t_{\nu}\right)\right) \alpha_{\kappa j}^{1}(t)=B_{j}\left(t, t_{\nu}\right)+O\left(1 /\left(\varepsilon(\nu-m)^{2}\right),\right. \\
\nu=s+1, s+2, \ldots, 2 m, \quad j=1,2, \ldots, n .
\end{array}
$$

Since Lemma 5.11 yields $\left\|Z_{1}(t, \xi)\right\|_{R^{n \times n}} \leq C / \varepsilon$, from (5.37) we then obtain

$$
\begin{aligned}
\sum_{\kappa=m+i_{0}}^{2 m-2}\left(\delta_{\nu \kappa+2}+\widehat{\Phi}_{\kappa j}\left(t_{\nu}\right)+\hat{\mu}_{\kappa j}\left(t_{\nu}\right)\right) \alpha_{\kappa j}^{1}(t) & =O(1 / \varepsilon), \\
\nu & =m+i_{0}+2, \ldots, s, \quad j=1,2, \ldots, n,
\end{aligned}
$$

in a similar way.

Let us consider (5.40), (5.41) as a system of linear algebraic equations with unknowns $\alpha_{\alpha j}^{1}(t)$. From Lemma 5.3 it follows that for sufficiently large $i_{0}$ the matrix of this system has its inverse matrix bounded uniformly in $\varepsilon$ and $m$. Therefore, since $\left\|G_{\varepsilon}(t, \xi)\right\|_{R^{n \times n}} \leq C / \varepsilon$, we have

$$
\max _{\nu, j ; t \in[-1,1]}\left\|\alpha_{\kappa j}^{1}(t)\right\|_{R^{n}} \leq C / \varepsilon .
$$

By virtue of Lemma 5.3 , the values $\mu_{\kappa j}\left(\hat{t}_{\nu}\right)$ and $\Phi_{\kappa j}(\hat{t})$ satisfy the relations

$$
\left|\hat{\mu}_{\kappa j}\left(t_{\nu}\right)\right| \leq C \min \left\{1 /(\kappa-m)^{3}, 1 /(\nu-m)^{3}\right\}, \quad \widehat{\Phi}_{\kappa j}\left(t_{\nu}\right)=O(1 /(\kappa-m)) \delta_{\nu \kappa+2} .
$$

Finally, transform the system (5.40) in the following way. Move the terms with number $\kappa=m+i_{0}, \ldots, s-2$ to the right-hand side and use the estimates (5.42), (5.43). As a result we get

$$
\sum_{\kappa=s-1}^{2 m-2} \delta_{\nu \kappa+2}\left(1+O(1 /(\kappa-m)) \alpha_{\kappa j}^{1}(t)=\beta_{j}\left(t, t_{\nu}\right)+O\left(1 /\left(\varepsilon(\nu-m)^{2}\right)\right.\right.
$$

for $\nu=s+1, s+2, \ldots, 2 m$. This is a system with a diagonal matrix. Solving this system, we have

$$
\alpha_{\kappa j}^{1}(t)=\beta_{j}\left(t, t_{\kappa+2}\right)\left(1+O(1 /(\kappa-m))+O\left(1 /\left(\varepsilon(\kappa-m)^{2}\right)\right)\right) .
$$

Hence (5.36) holds. The proof is completed.

\section{Properties of the functions $G_{\varepsilon} \lambda_{i j}(t)$}

The two statements below play an important role in the proof of Theorem 5 .

Lemma 6.1. The following formulas are valid:

$1^{\circ}$. For $-m \leq i \leq m-3, t \in\left[t_{s}, t_{s+1}\right]$, and $-2 m \leq s \leq 2 m-1$,

$$
\left\|G_{\varepsilon} \lambda_{i j}(t)\right\|_{R^{n}} \leq C m\left(\frac{1}{(1+|i-s|)^{2}}+\frac{1}{(n-|i|)^{3 / 2}}\right) .
$$

$2^{\circ}$. For $|i| \geq m+i_{0}, t \in\left[t_{s}, t_{s+1}\right]$, and $s \in\left[-2 m, m+i_{0}+1\right](i>0)$ or $s \in\left[-m-i_{0}-2,2 m-1\right](i<0)$,

$$
\left\|G_{\varepsilon} \lambda_{i j}(t)\right\|_{R^{n}} \leq \frac{C}{\varepsilon}\left(\frac{1}{(1+|i-s|)^{2}}+\frac{1}{(|i|-m)^{3 / 2}}\right) .
$$


Lemma 6.2. For $|i| \geq m+i_{0}, t \in\left[t_{s}, t_{s+1}\right],|s| \geq m+i_{0}+2$, and $i>s$ we have

$$
G_{\varepsilon} \lambda_{i j}(t)=\beta_{j}\left(t, t_{s}\right)\left(1+O\left(\frac{1}{|i|-m}\right)+\varepsilon^{-1} O\left(\frac{1}{(|i|-m)^{2}}+\frac{1}{(1+|i-s|)^{3 / 2}}\right)\right),
$$

where the $\beta_{j}(t, \xi)$ are defined on $[-1,1] \times[-1,1]$, and

$$
\left\|\partial^{q} \beta_{j}(t, \xi) / \partial \xi^{q}\right\|_{R^{n}} \leq \frac{C}{\varepsilon^{1+q}} \exp \left(-\nu_{0}|t-\xi| / \varepsilon\right), \quad q=0,1
$$

6.1. Proof of (6.1). Let $t \in\left[t_{s}, t_{s+1}\right]$. In accordance with (5.12), the relation $L_{i j}=O^{*}(1 / m)$ and Lemma 1.1 we have

$$
\begin{gathered}
\left\|G_{\varepsilon} \lambda_{i j}(t)\right\|_{R^{n}}=\left\|\int_{-1}^{1} G_{\varepsilon}(t, \xi) \lambda_{i j}(\xi) d \xi\right\|_{R^{n}}=\sum_{\nu=-2 m}^{2 m-1}\left\|\int_{t_{\nu}}^{t_{\nu+1}} G_{\varepsilon}(t, \xi) \lambda_{i j}(\xi) d \xi\right\|_{R^{n}} \\
\leq \frac{c}{\varepsilon} \sum_{i=-2 m}^{2 m-1} \frac{m}{(1+|i-m|)^{2}} \int_{t_{\nu}}^{t_{\nu+1}} \exp \left(-\nu_{0}|t-\xi| / \varepsilon\right) d \xi \\
\leq C m \sum_{\nu=-2 m}^{2 m-1} \frac{1}{(1+|i-m|)^{2}} \exp \left(-\nu_{0}\left|t-t_{\nu}\right| / \varepsilon\right)\left(1-\exp \left(-\nu_{0} h_{\nu} / \varepsilon\right) .\right.
\end{gathered}
$$

If $\nu$ changes from $-m$ to $m$, then all values $\exp \left(-\nu_{0}\left|t-t_{\nu}\right| / \varepsilon\right)$, except maybe a single one, would have been of order $O\left(\varepsilon^{2}\right)$ due to the condition $\varepsilon|\ln \varepsilon| \ll 1 / m$. If $|\nu| \geq m+1$, then

$$
1-\exp \left(-\nu_{0} h_{\nu} / \varepsilon\right) \leq C h_{\nu} / \varepsilon \leq C /(|\nu|-m) .
$$

From these facts we have

$$
\begin{aligned}
\left\|G_{\varepsilon} \lambda_{i j}(t)\right\|_{R^{n}} \leq \frac{C m}{(1+|i-m|)^{2}}+C m\left(\sum_{\nu=m+1}^{2 m-1} \frac{1}{(1+|i-\nu|)^{2}} \frac{\exp \left(-\nu_{0}\left|t-t_{\nu}\right| / \varepsilon\right)}{\nu-m}\right) \\
\quad+\sum_{\nu=-2 m}^{-m-1} \frac{1}{1 /(1+|i-\nu|)^{2}} \frac{\exp \left(-\nu_{0}\left|t-t_{\nu}\right| / \varepsilon\right)}{|\nu|-m} \\
\leq \frac{C m}{(1+|i-s|)^{2}}+C m\left(\sum_{\nu=m+1}^{2 m-1} \frac{1}{(\nu-i)^{3}(\nu-m)}+\sum_{\nu=-2 m}^{-m-1} \frac{1}{(|\nu|-m)(i-\nu)^{2}}\right) .
\end{aligned}
$$

Further,

$$
\begin{aligned}
& \sum_{\nu=m+1}^{2 m-1} \frac{1}{(\nu-i)^{2}(\nu-m)}=\sum_{\nu=m+1}^{2 m-i}(\cdots)+\sum_{\nu=2 m-i+1}^{2 m-1}(\cdots) \\
& \leq \frac{C \ln (m-i)}{(m-i)^{2}}+C \sum_{\nu=2 m-i}^{\infty} \frac{1}{(\nu-m)^{2}} \leq \frac{C}{(m-|i|)^{3 / 2}} .
\end{aligned}
$$

The second sum in (6.6) is estimated analogously. Thus from (6.6) we get

$$
\left\|G_{\varepsilon} \lambda_{i j}\right\|_{R^{n}} \leq C m\left(1 /(1+|i-s|)^{2}+1 /(m-|i|)^{2}\right) .
$$

This concludes the proof. 
6.2. Proof of (6.2). To be specific, let $i \geq m+i_{0}$ and $t \in\left[t_{s}, t_{s+1}\right] \subset\left[-1, t_{m+i_{0}+2}\right]$ (the other case is considered in a similar way). By virtue of (5.9), (5.10) and Lemma 5.1

$$
\begin{aligned}
\left\|G_{\varepsilon} \lambda_{i j}(t)\right\|_{R^{n}} & =\left\|\sum_{\nu=-2 m}^{2 m-1} \int_{t_{\nu}}^{t_{\nu+1}} G_{\varepsilon}(t, \xi) \lambda_{i j}(\xi) d \xi\right\|_{R^{n}} \\
& \leq \sum_{\nu=-2 m}^{2 m-1} \sum_{s=1}^{n} \frac{1}{L_{\nu s}}(1+|i-\nu|)^{2} \int_{t_{\nu}}^{t_{\nu+1}} \exp \left(-\nu_{0}|t-\xi| / \varepsilon\right) d \xi \\
& \leq \frac{C}{\varepsilon} \sum_{\nu=-2 m}^{2 m-1} \frac{1}{(1+|i-\nu|)^{2}} \max _{\xi \in\left[t_{\nu}, t_{\nu+1}\right]} \exp \left(-\nu_{0}|t-\xi| / \varepsilon\right) .
\end{aligned}
$$

First consider the case when $t \in\left[t_{-m-i_{0}-2}, t_{m+i_{0}+2}\right]$. Since $\varepsilon|\ln \varepsilon| \ll 1 / m$, due to Lemmas 5.1 and 2.1 we then have

$$
\max _{\xi \in\left[t_{\nu}, t_{\nu+1}\right]} \exp \left(-\nu_{0}|t-\xi| / \varepsilon\right) \leq\left\{\begin{aligned}
O\left(\varepsilon^{2}\right), & \text { for all } \nu \text { except maybe } \nu=s-1, \\
s, s+1, \text { if } t \in\left[t_{-m}, t_{m}\right], & \text { if } t \in\left[t_{m}, t_{m+i_{0}+2}\right], \\
O\left(1 /(1+|\nu-m|)^{2}\right), & \text { if } t \in\left[t_{-m-i_{0}-2}, t_{-m}\right] .
\end{aligned}\right.
$$

Therefore from Proposition 5.1 we have

$$
\begin{aligned}
& \frac{c}{\varepsilon} \sum_{\nu=-2 m}^{2 m-1} \frac{1}{(1+|i-m|)^{2}} \max _{\xi \in\left[t_{\nu}, t_{\nu+1}\right]} \exp \left(-\nu_{0}|t-\xi| / \varepsilon\right) \\
& =\frac{C}{\varepsilon}\left(\sum_{\nu=-2 m}^{-m-i_{0}} \frac{1}{\left.(1+|i-\nu|)^{2}(1+|\nu-m|)^{2}\right)}+\frac{1}{(1+|i-s|)^{2}}\right. \\
& \left.\quad+\sum_{\nu=m+i_{0}}^{2 m} \frac{1}{(1+|i-\nu|)^{2}}\right) \\
& \leq \frac{C}{\varepsilon}\left(\frac{1}{(1+|i-s|)^{2}}+\frac{1}{(1+|i-m|)^{2}}\right) .
\end{aligned}
$$

From (6.8) and (6.9) we have

$$
\left\|G_{\varepsilon} \lambda_{i j}(t)\right\|_{R^{n}} \leq \frac{C}{\varepsilon}\left(\frac{1}{(1+|i-s|)^{2}}+\frac{1}{(1+|i-m|)^{2}}\right),
$$

which yields the estimate (6.2).

Now let $t \in\left[-1, t_{-m-i_{0}-2}\right]$. In this case we have

$$
\left\|G_{\varepsilon} \lambda_{i j}(t)\right\|_{R^{n}} \leq\left\|\int_{-1}^{t_{-m}} G_{\varepsilon}(t, \xi) \lambda_{i j}(\xi) d \xi\right\|_{R^{n}}+\left\|\int_{t_{-m}}^{1} G_{\varepsilon}(t, \xi) \lambda_{i j}(\xi) d \xi\right\|_{R^{n}} .
$$

Since $\left|L_{i j}\right| \geq h_{2 m-1} \geq C m / \varepsilon$, by virtue of (5.11) one gets

$$
\left\|\int_{-1}^{t_{-m}} G_{\varepsilon}(t, \xi) \lambda_{i j}(\xi) d \xi\right\|_{R^{n}} \leq \frac{C}{m^{5 / 2}} \frac{m}{2} \int_{-1}^{t_{-m-1}}\left\|G_{\varepsilon}(t, \xi)\right\|_{R^{n \times n}} d \xi \leq \frac{C}{\varepsilon m^{5 / 2}} .
$$

The second term in (6.10) may be estimated from the inequality

$$
\left\|G_{\varepsilon}(t, \xi)\right\|_{R^{n \times n}} \leq C / \varepsilon \exp \left(\nu_{0}(\xi-t) / \varepsilon\right) \leq C \varepsilon^{2}
$$


for $g \in\left[t_{-m}, 1\right]$ (compare (6.8)). This concludes the proof.

6.3. Proof of Lemma 6.2. To be specific, let $i \geq m+i$ and $s \geq m+i_{0}+2$. In the case $s \leq i+2$ we have

$$
G_{\varepsilon} \lambda_{i j}(t)=\int_{-1}^{1}\left(G_{\varepsilon}(t, \xi)-Z_{1}(t, \xi)\right) \lambda_{i j}(\xi) d \xi+\int_{-1}^{1} Z_{1}(t, \xi) \lambda_{i j}(\xi) d \xi .
$$

Due to Lemma 5.13,

$$
\left\|\int_{-1}^{1}\left(G_{\varepsilon}(t, \xi)-Z_{1}(t, \xi)\right) \lambda_{i j}(\xi) d \xi\right\|_{R^{n}} \leq \frac{C}{\varepsilon}\left(\frac{1}{(|i|-m)^{3 / 2}}+\frac{1}{(1+|i-s|)^{3 / 2}}\right) .
$$

Further, according to the definition of $\lambda_{i j}(t)$ and (5.35), (5.36),

$$
\int_{-1}^{1} Z_{1}(t, \xi) \lambda_{i j}(\xi) d \xi=\alpha_{i j}^{1}(t)=\beta_{i j}\left(t, t_{i+2}\right)\left(1+O(1 /(|i|-m))+O\left(1 /\left(\varepsilon(|i|-m)^{2}\right) .\right.\right.
$$

The relations (6.11), (6.13) and (5.17) imply formulas (6.3)-(6.4). Lemma 6.2 is proved.

\section{Proof of Theorem 5}

7.1. Galerkin projection. Let $D=\left\{x \in\left(C^{1}[-1,1]\right)^{n}: x^{1}(-1)=\cdots=x^{k}(-1)=\right.$ $\left.x^{k+1}(1)=\cdots=x^{n}(1)=0\right\}$ be the domain of definition of the operator $L_{\varepsilon}$. Let $x \in D$ and $f=L_{\varepsilon} x$. Then $x$ is the solution of the corresponding problem (1.1)-(1.2) for $d=f$.

Let $P_{m}$ be the orthogonal projection of $\left(L_{2}[-1,1]\right)^{n}$ onto $F(\varepsilon, m)$. It is easy to show that the Galerkin problem (2.7) for $d=f$ is equivalent to the problem $P_{m} L_{\varepsilon} x_{m}=P_{m} f$ or $L_{\varepsilon} x_{m}=P_{m} f$. Hence $x_{m}=G_{\varepsilon} P_{m} f=G_{\varepsilon} P_{m} L_{\varepsilon} x$. The operator $Q_{m}=G_{\varepsilon} P_{m} L_{\varepsilon}: D \rightarrow E$ is called the Galerkin projection (see [4]). Obviously $Q_{m}^{2}=Q_{m}$.

Error estimates for the solution of problem (2.7) are closely connected to an estimate of the norm of projection $Q_{m}$. Namely, the following statement is true.

Lemma 7.1. The following estimates hold:

$$
\left\|x-Q_{m} x\right\|_{\infty} \leq\left(1+\left\|Q_{m}\right\|_{\infty}\right)\left\|x-\tilde{x}_{m}\right\|_{\infty},
$$

where

$$
\left\|Q_{m}\right\|_{\infty}=\left\|Q_{m}\right\|_{L_{\infty} \rightarrow L_{\infty}}=\sup _{x \in D:\|x\|_{\infty} \leq 1}\left\|Q_{m} x\right\|_{\infty}
$$

and $\tilde{x}$ is the best approximation of $x$ in $E$ in the sense of $L_{\infty}[-1,1]$.

Since $Q_{m} \tilde{x}_{m}=x_{m}$, the proof is evident.

Since $x_{\varepsilon}$ satisfies (1.5), one can easily obtain $\left\|x_{\varepsilon}-\hat{x}_{\varepsilon}\right\|_{\infty} \leq C / m^{3}$ through de Boor's approximation theorem analogously to Lemma 5.2. Hence, by means of Lemma 7.1, we conclude that it is sufficient to prove the estimate

$$
\left\|Q_{m}\right\|_{\infty} \leq C
$$

uniformly in $\varepsilon$ and $m$. 
7.2. Representation of Galerkin projection and preliminary estimates. Considering

$$
Q_{m}=G_{\varepsilon} P_{m} L_{\varepsilon} \quad \text { and } \quad P_{m} y=\sum_{j=1}^{n} \sum_{i=-2 m-2}^{2 m-2}\left(y, N_{i j}\right) \lambda_{i j}(t),
$$

we have

$$
Q_{m} x=\sum_{j=1}^{n} \sum_{i=-2 m-2}^{2 m-2}\left(L_{\varepsilon} x, N_{i j}\right) G_{\varepsilon} \lambda_{i j}(t) .
$$

Since we need to bound the norm of $Q_{m}$, we shall further consider $\|x\|_{\infty} \leq 1$. Integrating (7.2) by parts, we get rid of the derivative of the function $x$. As a result we obtain

$$
Q_{m} x=\sum_{i, j}\left[\left(x_{\varepsilon}, L_{\varepsilon}^{*} N_{i j}\right)+\varepsilon \phi_{\varepsilon}\left(x(-1), N_{i j}(-1)\right)+\varepsilon \psi_{\varepsilon}\left(x(1), N_{i j}(1)\right)\right] G_{\varepsilon} \lambda_{i j}(t),
$$

where $\phi_{\varepsilon}$ and $\psi_{\varepsilon}$ are bilinear functionals, bounded uniformly on $\varepsilon$, by means of which the terms outside the integral are expressed.

From estimates of the function $N_{i j}$ (see Lemma 3.4) we obtain

$$
\begin{gathered}
\left|\phi_{\varepsilon}\left(x(-1), N_{i j}(-1)\right) \leq C, \quad\right| \psi_{\varepsilon}\left(x(1), N_{i j}(1)\right) \mid \leq C, \quad i=-2 m-2,2 m-2, \\
\quad\left|\phi_{\varepsilon}\left(x(-1), N_{i j}(-1)\right)\right| \leq C /(2 m+2-|i|)^{3}, \\
\left|\psi_{\varepsilon}\left(x(1), N_{i j}(1)\right)\right| \leq C /(2 m+2-|i|)^{3}, \quad i \neq-2 m-2,2 m-2 .
\end{gathered}
$$

Due to Lemma $5.9,\left\|\varepsilon G_{\varepsilon} \lambda_{i j}\right\| \leq C$. This fact and (7.4), (7.5) lead to

$$
\left\|\sum_{i, j} \varepsilon\left(\phi_{\varepsilon}\left(x(-1), N_{i j}(-1)\right)+\psi_{\varepsilon}\left(x(1), N_{i j}(1)\right)\right) G_{\varepsilon} \lambda_{i j}\right\|_{\infty} \leq C .
$$

From (7.3), (7.4) it follows that, if the bound

$$
\left\|\sum_{j=1}^{n} \sum_{i=-2 m-2}^{2 m-2}\left(x, L_{\varepsilon}^{*} N_{i j}\right) G_{\varepsilon} \lambda_{i j}(t)\right\|_{\infty} \leq C
$$

holds for arbitrary $x \in\left(L_{\infty}[-1,1]\right)^{n}$ with $\|x\|_{\infty} \leq 1$, then it would be sufficient to prove the estimate (7.1).

7.3. Proof of (7.7). We first notice that, by (5.6) and (5.16), every term in the sum (7.7) is bounded uniformly in $\varepsilon$ and $m$. Thus it is sufficient to bound each of the expressions

$$
I_{1}=\left\|\sum_{i=-m}^{m-3}\left(x, L_{\varepsilon}^{*} N_{i j}\right) G_{\varepsilon} \lambda_{i j}(t)\right\|_{\infty}
$$

and

$$
I_{2}=\left\|\sum_{i=m+i_{0}}^{2 m-2}\left(x, L_{\varepsilon}^{*} N_{i j}\right) G_{\varepsilon} \lambda_{i j}(t)\right\|_{\infty}
$$


(the estimate of the sum $\sum_{i=-2 m-2}^{-m-i_{0}}$ can be obtained through a similar argument as for $(7.9))$.

We first consider $I_{1}$. Let $t \in\left[t_{s}, t_{s+1}\right]$. According to (6.1) and (5.6) we have

$$
\begin{aligned}
\left\|\sum_{i=-m}^{m-3}\left(x, L_{\varepsilon} N_{i j}, G_{\varepsilon} \lambda_{i j}(t)\right)\right\|_{R^{n}} & \leq \frac{C}{m} \sum_{i=-m}^{m-3}\left\|G_{\varepsilon} \lambda_{i j}(t)\right\|_{R^{n}} \\
& \leq C \sum_{i=-m}^{m} \frac{1}{(1+|i-s|)^{3 / 2}} \leq C,
\end{aligned}
$$

and hence

$$
I_{1} \leq C
$$

Now let us bound $I_{2}$. If $t \in\left[t_{s}, t_{s+1}\right] \subset\left[-1, t_{m+i_{0}+2}\right]$, then $I_{2}$ can be bounded in the same way as $I_{1}$ by (5.6) and (6.2). Let $t \in\left[t_{s}, t_{s+1}\right] \subset\left[t_{m+i_{0}+2}, 1\right]$. In this case by (7.6) and (6.3)-(6.4) we have

$$
\begin{aligned}
& \| \sum_{i=m+i_{0}}^{2 m-2}\left(x, L_{\varepsilon}^{*} N_{i j}\right)\left(\beta _ { j } ( t , T _ { i + 2 } ) \left(1+O\left(\frac{1}{i-m}\right)+O\left(\frac{1}{\varepsilon(i-m)^{2}}\right)\right.\right. \\
& \left.\left.+\frac{1}{\varepsilon(1+|i-s|)^{3 / 2}}\right)\right) \|_{R^{n}} \\
& \leq C \sum_{i=m+i_{0}}^{2 m-2}\left(\frac{1}{(i-m)^{2}}+\frac{1}{(1+|i-m|)^{3 / 2}}\right) \\
& +C \varepsilon\left\|\sum_{i=m+i_{0}}^{2 m-2} \frac{\beta_{j}\left(t, t_{i+2}\right)}{i-m}\right\|_{R^{n}}+\left\|\sum_{i=m+i_{0}}^{2 m-2}\left(x, L_{\varepsilon}^{*} N_{i j}\right) \beta_{j}\left(t, t_{i+2}\right)\right\|_{R^{n}} \\
& \leq C+C\left|\sum_{i=m+i_{0}}^{2 m-2} \frac{1}{i-m} \exp \left(-\nu_{0}\left|t-t_{i+2}\right| / \varepsilon\right)\right|+\left\|\sum_{i=m+i_{0}}^{2 m-2}\left(x, L_{\varepsilon}^{*} N_{i j}\right) \beta_{j}\left(t, t_{i+2}\right)\right\|_{R^{n}} .
\end{aligned}
$$

Further, due to Lemma 5.1, we get

$$
\begin{aligned}
& \left|\sum_{i=m+i_{0}}^{2 m-2} \frac{1}{i-m} \exp \left(-\nu_{0}\left|t-t_{i+2}\right| / \varepsilon\right)\right| \\
& \quad \leq C\left(\sum_{i=m+i_{0}}^{s-2} \frac{(i-m)^{2}}{(s-m)^{3}}+\sum_{i=s-1}^{2 m-2} \frac{(s-m)^{3}}{(i-m)^{4}}\right) \leq C .
\end{aligned}
$$

Thus, to complete the estimation of $I_{2}$ it is sufficient to bound the last expression in (7.11). For every $j=1,2, \ldots, n$

$$
\begin{aligned}
& \left\|\sum_{i=m+i_{0}}^{2 m-2}\left(x, L_{\varepsilon}^{*} N_{i j}\right) \beta_{j}\left(t, t_{i+2}\right)\right\|_{R^{n}} \leq\left\|\sum_{i=m+i_{0}}^{s-2}\left(x, L_{\varepsilon}^{*} N_{i j}\right) \beta_{j}\left(t, t_{i+2}\right)\right\|_{R^{n}} \\
& +\left\|\sum_{i=s-1}^{2 m-2}\left(x, L_{\varepsilon}^{*} N_{i j}\right) \beta_{j}\left(t, t_{i+2}\right)\right\|_{R^{n}} .
\end{aligned}
$$


To bound each sum in the right-hand side of (7.12), use Abel's transformation

$$
\sum_{i=p}^{q}\left(A_{i+1}-A_{i}\right) b_{i}=A_{q+1} b_{q}-A_{p} b_{p}-\sum_{i=p}^{q-1} A_{i}\left(b_{i+1}-b_{i}\right) .
$$

To estimate the first sum put $b_{i}=\beta_{j}\left(t, t_{i+2}\right)$; then

$$
A_{i}=-\sum_{\nu=i}^{s-2}\left(x, L_{\varepsilon}^{*} N_{\nu j}\right) \quad\left(i=m+i_{0}, m+i_{0}+1, \ldots, s-2 ; p=m+i_{0}, q=s\right) .
$$

Then, by virtue of (7.12) and (7.13),

$$
\begin{aligned}
& \left\|\sum_{i=m+i_{0}}^{s-2}\left(x, L_{\varepsilon}^{*} N_{i j}\right) \beta_{j}\left(t, t_{i+2}\right)\right\|_{R^{n}} \\
& \leq\left\|\left(x, L_{\varepsilon}^{*} \sum_{\nu=m+i_{0}}^{s-2} N_{\nu j}\right) \beta_{j}\left(t, t_{m+i_{0}+2}\right)\right\|_{R^{n}}+\left\|\left(x, L_{\varepsilon}^{*} N_{s-2 j}\right) \beta_{j}\left(t, t_{s}\right)\right\|_{R^{n}} \\
& \quad+\left\|\sum_{i=m+i_{0}}^{s-3}\left(x, L_{\varepsilon}^{*} \sum_{\nu=i}^{s+2} N_{\nu j}\right)\left(\beta_{j}\left(t, t_{i+2}\right)-\beta_{j}\left(t, t_{i+1}\right)\right)\right\|_{R^{n}} .
\end{aligned}
$$

Further, due to Lemmas 5.1, 5.5 and estimates (6.4),

$$
\begin{aligned}
& \left\|\left(x, L_{\varepsilon}^{*} \sum_{\nu=m+i_{0}}^{s-2} N_{\nu j}\right) \beta_{j}\left(t, t_{m+i_{0}+2}\right)\right\|_{R^{n}} \\
& \leq \frac{C}{\varepsilon}\left|\left(x, L_{\varepsilon}^{*} \sum_{\nu=m+i_{0}}^{s-2} N_{\nu j}\right) \exp \left(\nu_{0}\left(t_{m+i_{0}+2}-t\right) / \varepsilon\right)\right| \\
& \leq \varepsilon \ln (s-m) \leq C, \\
& \left\|\sum_{i=m+i_{0}}^{s-3}\left(x, L_{\varepsilon}^{*} \sum_{\nu=i}^{s-2} N_{\nu j}\right)\left(\beta_{j}\left(t, t_{i+2}\right)-\beta_{j}\left(t, t_{i+1}\right)\right)\right\|_{R^{n}} \\
& \left.\leq C \sum_{s j}\right) \beta_{j}\left(t, t_{s}\right)\left\|_{\infty} \leq C\right\| x \|_{\infty} \leq C, \\
& \left.\leq C\|x\|_{\infty}, \quad x, L_{\varepsilon}^{*} \sum_{\nu=i}^{s-2} N_{\nu j}\right) \mid h_{i}\left(1 / \varepsilon^{2}\right) \exp \left(-\nu_{0}\left|t-t_{i+2}\right| / \varepsilon\right) \|_{R^{n}} \\
& \left\|\sum_{i=m+i_{0}}^{s-3} \frac{\ln ((s-m-2) /(i-m))}{i-m} \exp \left(-\nu_{0}\left|t-t_{i+2}\right| / \varepsilon\right)\right\|_{R^{n}} \\
& \quad \leq \frac{C}{(s-m)^{3}} \sum_{i=m+i_{0}}^{s-3} \ln ((s-m-2) /(i-m))(i-m)^{2} \leq C
\end{aligned}
$$

(the last step in (7.17) is made by means of Proposition 5.2). 
From (7.14)-(7.17) the estimate for the first sum in (7.12) follows. The second sum is estimated in the same way by setting

$$
\begin{gathered}
b_{i}=\beta_{j}\left(t, t_{i+2}\right), \quad A_{i}=\sum_{\nu=s-1}^{i-1}\left(x, L_{\varepsilon}^{*} N_{i j}\right), \\
i=s-1, s, \ldots, 2 m-1, \quad p=s-1, \quad q=2 m-2,
\end{gathered}
$$

and making calculations analogous to (7.14)-(7.17). This completes the proof of Theorem 5 .

\section{NUMERICAL EXAMPLES}

To confirm the theoretical results we consider the following examples.

\section{Example 1.}

$$
\begin{gathered}
\varepsilon x^{\prime}=\left(\begin{array}{cc}
-1 & 1 \\
3 & 1
\end{array}\right) x+\left(\begin{array}{c}
1 \\
-1
\end{array}\right), \quad x=\left(x^{1}, x^{2}\right)^{T}, \\
x^{1}(-1)=x^{2}(1)=0 .
\end{gathered}
$$

The exact solution of the problem (8.1)-(8.2) may be written in the form

$$
\begin{aligned}
& x^{1}(t)=C_{1} \exp (2 t / \varepsilon)-C_{2} \exp (-2 t / \varepsilon)+0.5, \\
& x^{2}(t)=3 C_{1} \exp (2 t / \varepsilon)+3 C_{2} \exp (-2 t / \varepsilon)-0.5,
\end{aligned}
$$

where

$$
\begin{aligned}
& C_{1}=(\exp (2 / \varepsilon)-\exp (-2 / \varepsilon)) /(2(3 \exp (4 / \varepsilon)+\exp (-4 / \varepsilon))), \\
& C_{2}=(3 \exp (2 / \varepsilon)-\exp (-2 / \varepsilon)) /(2(3 \exp (4 / \varepsilon)+\exp (-4 / \varepsilon))) .
\end{aligned}
$$

Example 2 ([23]).

$$
\begin{gathered}
\varepsilon x^{\prime}=A(t, \lambda) x+f(t, \varepsilon, \lambda), \quad x=\left(x^{1}, x^{2}\right)^{T}, \quad x^{-1}(-1)=x^{2}(1)=0, \\
A(t, \lambda)=E(t, \lambda)\left(\begin{array}{cc}
-1 & 0 \\
0 & 2
\end{array}\right) E^{-1}(t, \lambda) \\
E(t, \lambda)=E^{-1}(t, \lambda)=\left(\begin{array}{cc}
\sin \lambda t & \cos \lambda t \\
\cos \lambda t & -\sin \lambda t
\end{array}\right) .
\end{gathered}
$$

Example 2 differs from the example considered in [23] only by inessential variation of the boundary conditions. Analogously with [23] (see (7.1)) we write the solution of the problem (8.3), (8.4) in the form

$$
x(t)=E(t, \lambda) \Phi(t, \lambda) s+x_{p}(t)
$$

where $E(t, \lambda) \Phi(t, \lambda)$ is a fundamental matrix of a homogenous system (8.3) (see [23]) and $x_{p}(t)=(\exp (t), \exp (-t))^{T}$. A constant vector $s$ should be chosen in such a way that $x(t)$ satisfies the boundary condition (8.4). As in [23], we set $\lambda=\pi / 4$.

The results of the numerical computations for Examples 1 and 2 are presented in Tables 1-6. For $\varepsilon$ and $m$ the corresponding cell contains the quantity

$e_{e, m}=\max _{i=1,2} \max _{-2 m \leq j \leq 2 m} \max _{0 \leq k \leq 10}\left|x_{m}^{i}\left(t_{j}+k\left(t_{j+1}-t_{j}\right) / 10\right)-x_{\varepsilon}\left(t_{j}+k\left(t_{j+1}-t_{j}\right) / 10\right)\right|$ in the upper part of the cell and the observed rate

$$
v_{\varepsilon, m}=\log _{2}\left(e_{\varepsilon, m / 2} / e_{\varepsilon, m}\right)
$$

in the lower part of the cell. 
The numerical results for the collocation method are given in Tables 1-4. Tables 1 and 2 contain the results for Examples 1 and 2, respectively, for the algorithm in cases $(\alpha)$ and $(\beta)$; the analogous results for case $(\gamma)$ are presented in Tables 3 and (4). The numerical results for the Galerkin method are presented in Tables 5 and 6. The symbol "-" means the observed rate becomes negative.

Our numerical examples show that the observed rates of convergence for the collocation method tends to 2 uniformly in $\varepsilon$ and $m$ with $1 /(\varepsilon m) \leq$ const in cases $(\alpha)$ and $(\beta)$, and in $\varepsilon$ and $m$ with $\varepsilon m \leq$ const in case $(\gamma)$. Note that, for $\varepsilon \ll 1 / m$ in cases $(\alpha)$ and $(\beta)$, and for $1 / m \ll \varepsilon$ in case $(\gamma)$, errors of evaluation strictly grow. This means that different values $\varepsilon / m$ need to be applied in different algorithms.

The observed rate for the Galerkin method is close to 3 uniformly for small $\varepsilon$.

TABle 1

\begin{tabular}{|c|c|c|c|c|c|c|}
\hline $\begin{array}{l}\backslash m \\
\varepsilon \backslash\end{array}$ & 4 & 8 & 16 & 32 & 64 & 128 \\
\hline $2 E-1$ & $3.51 E-2$ & $\frac{7.35 E-3}{2.25}$ & $\frac{1.73 E-3}{2.08}$ & $\frac{4.24 E-4}{2.03}$ & $\frac{1.05 E-4}{2.01}$ & $\frac{2.62 E-5}{2.007}$ \\
\hline $1 E-1$ & $4.68 E-2$ & $\frac{9.33 E-3}{2.33}$ & $\frac{2.19 E-3}{2.09}$ & $\frac{5.38 E-4}{2.03}$ & $\frac{1.33 E-4}{2.01}$ & $\frac{3.31 E-5}{2.008}$ \\
\hline $3 E-2$ & $5.60 E-2$ & $\frac{1.08 E-2}{2.38}$ & $\frac{2.58 E-2}{2.06}$ & $\frac{6.27 E-4}{2.04}$ & $\frac{1.55 E-4}{2.02}$ & $\frac{3.85 E-5}{2.008}$ \\
\hline $1 E-2$ & $5.88 E-2$ & $\frac{1.12 E-2}{2.40}$ & $\frac{2.69 E-3}{2.05}$ & $\frac{6.54 E-4}{2.04}$ & $\frac{1.61 E-4}{2.02}$ & $\frac{4.01 E-5}{2.009}$ \\
\hline $1 E-3$ & $\underline{1.71 E-1}$ & $\frac{1.13 E-2}{3.92}$ & $\frac{2.74 E-3}{2.05}$ & $\frac{6.66 E-4}{2.04}$ & $\frac{1.64 E-4}{2.02}$ & $\frac{4.08 E-5}{2.009}$ \\
\hline $1 E-4$ & $\underline{1.84 E+0}$ & $\frac{1.14 E-2}{7.34}$ & $\frac{2.75 E-3}{2.05}$ & $\frac{6.67 E-4}{2.04}$ & $\frac{1.65 E-4}{2.02}$ & $\frac{4.09 E-5}{2.009}$ \\
\hline $1 E-5$ & $\underline{1.88 E+1}$ & $\frac{8.54 E-2}{7.79}$ & $\frac{2.75 E-3}{4.96}$ & $\frac{6.67 E-4}{2.04}$ & $\frac{1.65 E-4}{2.02}$ & $\frac{2.009}{2.009}$ \\
\hline $1 E-6$ & $\underline{1.91 E+2}$ & $\frac{8.67 E-1}{7.78}$ & $\frac{6.04 E-3}{7.17}$ & $\frac{6.67 E-4}{3.13}$ & $\frac{1.65 E-4}{2.02}$ & $\frac{4.09 E-5}{2.009}$ \\
\hline
\end{tabular}


TABLE 2

\begin{tabular}{|c|c|c|c|c|c|c|}
\hline $\begin{array}{l}\backslash m \\
\varepsilon \backslash\end{array}$ & 4 & 8 & 16 & 32 & 64 & 128 \\
\hline $2 E-1$ & $3.51 E-2$ & $\frac{7.35 E-3}{2.29}$ & $\frac{1.73 E-3}{2.08}$ & $\frac{4.24 E-4}{2.03}$ & $\frac{2.39 E-4}{0.83}$ & $\frac{4.51 E-2}{-}$ \\
\hline $1 E-1$ & $4.82 E-2$ & $\frac{9.33 E-3}{2.33}$ & $\frac{2.19 E-3}{2.09}$ & $\frac{5.38 E-4}{2.03}$ & $\frac{1.33 E-4}{2.01}$ & $\frac{3.31 E-5}{2.008}$ \\
\hline $3 E-2$ & $5.60 E-2$ & $\frac{1.08 E-2}{2.23}$ & $\frac{2.58 E-3}{2.06}$ & $\frac{6.27 E-4}{2.04}$ & $\frac{1.55 E-4}{2.02}$ & $\frac{3.85 E-5}{2.008}$ \\
\hline $1 E-2$ & $5.89 E-2$ & $\frac{1.12 E-2}{2.40}$ & $\frac{2.69 E-3}{2.05}$ & $\frac{6.54 E-4}{2.04}$ & $\frac{1.61 E-4}{2.02}$ & $\frac{4.01 E-5}{2.009}$ \\
\hline $1 E-3$ & $6.02 E-2$ & $\frac{1.13 E-2}{2.41}$ & $\frac{2.75 E-3}{2.05}$ & $\frac{6.66 E-4}{2.04}$ & $\frac{1.64 E-4}{2.02}$ & $\frac{4.08 E-5}{2.009}$ \\
\hline $1 E-4$ & $6.03 E-2$ & $\frac{1.14 E-2}{2.41}$ & $\frac{2.75 E-3}{2.05}$ & $\frac{6.67 E-4}{2.04}$ & $\frac{1.65 E-4}{2.02}$ & $\frac{4.09 E-5}{2.009}$ \\
\hline $1 E-5$ & $6.03 E-2$ & $\frac{1.14 E-2}{2.41}$ & $\frac{2.75 E-3}{2.05}$ & $\frac{6.67 E-4}{2.04}$ & $\frac{1.65 E-4}{2.02}$ & $\frac{4.09 E-5}{2.009}$ \\
\hline $1 E-6$ & $6.03 E-2$ & $\frac{1.14 E-2}{2.41}$ & $\frac{2.75 E-3}{2.05}$ & $\frac{6.67 E-4}{2.04}$ & $\frac{1.65 E-4}{2.02}$ & $\frac{4.09 E-5}{2.009}$ \\
\hline
\end{tabular}

TABLE 3

\begin{tabular}{|c|c|c|c|c|c|c|}
\hline $\begin{array}{l}\backslash m \\
\varepsilon \backslash\end{array}$ & 4 & 8 & 16 & 32 & 64 & 128 \\
\hline $2 E-1$ & $3.74 E-2$ & $\frac{8.23 E-3}{2.18}$ & $\frac{2.03 E-3}{2.02}$ & $\frac{5.00 E-4}{2.02}$ & $\frac{1.24 E-4}{2.01}$ & $\frac{3.10 E-5}{2.004}$ \\
\hline $1 E-1$ & $4.10 E-2$ & $\frac{8.46 E-3}{2.28}$ & $\frac{2.07 E-3}{2.03}$ & $\frac{5.06 E-4}{2.03}$ & $\frac{1.25 E-4}{2.01}$ & $\frac{3.12 E-5}{2.006}$ \\
\hline $3 E-2$ & $4.33 E-2$ & $\frac{8.44 E-3}{2.36}$ & $\frac{2.05 E-3}{2.04}$ & $\frac{4.99 E-4}{2.04}$ & $\frac{1.23 E-4}{2.02}$ & $\frac{3.06 E-5}{2.008}$ \\
\hline $1 E-2$ & $4.40 E-2$ & $\frac{8.39 E-3}{2.41}$ & $\frac{2.03 E-3}{2.03}$ & $\frac{4.94 E-4}{2.04}$ & $\frac{1.22 E-4}{2.02}$ & $\frac{3.03 E-5}{2.009}$ \\
\hline $1 E-3$ & $\underline{1.76 E+0}$ & $\frac{8.37 E-3}{7.71}$ & $\frac{2.02 E-3}{2.05}$ & $\frac{4.91 E-4}{2.04}$ & $\frac{1.21 E-4}{2.02}$ & $\frac{3.01 E-5}{2.009}$ \\
\hline $1 E-4$ & $1.92 E+2$ & $\frac{9.38 E-1}{7.68}$ & $\frac{1.27 E-3}{6.02}$ & $\frac{4.91 E-4}{4.69}$ & $\frac{1.21 E-4}{2.02}$ & $\frac{3.01 E-5}{2.009}$ \\
\hline $1 E-5$ & $2.00 E+4$ & $\frac{1.01 E+2}{7.63}$ & $\frac{1.34 E+0}{6.23}$ & $\frac{1.96 E-2}{6.10}$ & $\frac{3.27 E-4}{5.90}$ & $\frac{3.01 E-5}{3.44}$ \\
\hline $1 E-6$ & $\infty$ & $\frac{1.04 E+4}{\infty}$ & $\frac{1.39 E+2}{6.23}$ & $\frac{2.12 E+0}{6.03}$ & $\frac{3.29 E-2}{6.01}$ & $\frac{5.16 E-5}{9.23}$ \\
\hline
\end{tabular}


TABLE 4

\begin{tabular}{|c|c|c|c|c|c|c|}
\hline \begin{tabular}{c}
$\backslash m$ \\
\multicolumn{1}{|c|}{4}
\end{tabular} & 4 & 16 & 32 & 64 & 128 \\
\hline $2 E-1$ & $\frac{3.42 E-2}{2.59 E-2}$ & $\frac{4.68 E-2}{-}$ & $\frac{6.68 E-2}{-}$ & $\frac{1.19 E-1}{-}$ & $\frac{1.64 E+0}{-}$ \\
\hline $1 E-1$ & $\frac{3.97 E-2}{8.40 E-3}$ & $\frac{3.07 E-3}{2.24}$ & $\frac{4.45 E-3}{-}$ & $\frac{5.29 E-3}{-}$ & $\frac{5.98 E-3}{-}$ \\
\hline $3 E-2$ & $\frac{4.29 E-2}{2.45}$ & $\frac{8.43 E-3}{2.35}$ & $\frac{2.05 E-3}{2.04}$ & $\frac{4.99 E-4}{2.04}$ & $\frac{1.23 E-4}{2.02}$ & $\frac{7.06 E-5}{0.80}$ \\
\hline $1 E-2$ & $\frac{4.35 E-2}{1 E-3}$ & $\frac{8.39 E-3}{2.38}$ & $\frac{2.03 E-3}{2.05}$ & $\frac{4.94 E-4}{2.04}$ & $\frac{1.21 E-4}{2.02}$ & $\frac{3.03 E-5}{2.009}$ \\
\hline $1 E-4$ & $\frac{4.46 E-2}{2.48 E-2}$ & $\frac{8.37 E-3}{2.42}$ & $\frac{2.02 E-3}{2.05}$ & $\frac{4.91 E-4}{2.04}$ & $\frac{1.21 E-4}{2.02}$ & $\frac{3.01 E-5}{2.009}$ \\
\hline $1 E-5$ & $\frac{8.48 E-2}{2.42}$ & $\frac{2.02 E-3}{2.05}$ & $\frac{4.91 E-4}{2.04}$ & $\frac{1.21 E-4}{2.02}$ & $\frac{3.01 E-5}{2.009}$ \\
\hline $1 E-6$ & $\frac{8.46 E-3}{2.42}$ & $\frac{2.02 E-3}{2.05}$ & $\frac{4.91 E-4}{2.04}$ & $\frac{1.21 E-4}{2.02}$ & $\frac{3.01 E-5}{2.009}$ \\
\hline
\end{tabular}

TABLE 5

\begin{tabular}{|c|c|c|c|c|c|c|}
\hline $\begin{array}{c}\backslash m \\
\varepsilon\end{array}$ & 4 & 8 & 16 & 32 & 64 & 128 \\
\hline $2 E-1$ & $\frac{1.18 E-3}{2 E}$ & $\frac{1.49 E-4}{2.99}$ & $\frac{1.85 E-5}{3.01}$ & $\frac{2.28 E-6}{3.02}$ & $\frac{3.12 E-7}{2.87}$ & $\frac{4.61 E-8}{2.66}$ \\
\hline $1 E-1$ & $\frac{1.73 E-3}{2.13 E-4}$ & $\frac{2.66 E-5}{3.02}$ & $\frac{3.32 E-6}{3.00}$ & $\frac{4.08 E-7}{3.02}$ & $\frac{5.21 E-8}{3.23}$ \\
\hline $3 E-2$ & $\frac{2.66 E-3}{2.88 E-4}$ & $\frac{3.47 E-5}{3.21}$ & $\frac{4.25 E-6}{3.05}$ & $\frac{5.26 E-7}{3.01}$ & $\frac{6.47 E-8}{3.02}$ \\
\hline $1 E-2$ & $\frac{3.62 E-3}{1 E-3}$ & $\frac{3.88 E-4}{3.22}$ & $\frac{4.28 E-5}{3.18}$ & $\frac{4.78 E-6}{3.16}$ & $\frac{5.76 E-7}{3.05}$ & $\frac{7.05 E-8}{3.03}$ \\
\hline $1 E-4$ & $\frac{6.15 E-3}{3}$ & $\frac{7.40 E-4}{3.05}$ & $\frac{8.82 E-5}{3.07}$ & $\frac{1.04 E-5}{3.08}$ & $\frac{1.21 E-6}{3.10}$ & $\frac{1.39 E-7}{3.12}$ \\
\hline $1 E-5$ & $\frac{6.76 E-3}{3.21 E-3}$ & $\frac{8.25 E-4}{3.03}$ & $\frac{1.00 E-4}{3.04}$ & $\frac{1.22 E-5}{3.04}$ & $\frac{1.46 E-6}{3.06}$ & $\frac{1.75 E-7}{3.06}$ \\
\hline $1 E-6$ & $\frac{7.18 E-3}{3.83 E-5}$ & $\frac{8.83 E-4}{3.02}$ & $\frac{1.08 E-4}{3.03}$ & $\frac{1.33 E-5}{3.02}$ & $\frac{1.62 E-6}{3.04}$ & $\frac{1.98 E-7}{3.03}$ \\
\hline
\end{tabular}


TABLE 6

\begin{tabular}{|c|c|c|c|c|c|c|}
\hline $\begin{array}{l}\backslash m \\
\varepsilon \backslash\end{array}$ & 4 & 8 & 16 & 32 & 64 & 128 \\
\hline $2 E-1$ & $\underline{5.66 E-3}$ & $\frac{5.80 E-4}{3.29}$ & $\frac{9.53 E-5}{2.61}$ & $\frac{1.21 E-5}{2.98}$ & $\frac{1.52 E-6}{2.99}$ & $\frac{2.92 E-7}{2.38}$ \\
\hline $1 E-1$ & $\underline{7.31 E-3}$ & $\frac{9.58 E-4}{2.93}$ & $\frac{1.23 E-4}{2.96}$ & $\frac{1.56 E-5}{2.98}$ & $\frac{1.96 E-6}{2.99}$ & $\frac{2.46 E-7}{2.99}$ \\
\hline $3 E-2$ & $8.56 E-3$ & $\frac{1.13 E-3}{2.92}$ & $\frac{1.43 E-4}{2.98}$ & $\frac{1.82 E-5}{2.97}$ & $\frac{2.28 E-6}{3.00}$ & $\frac{2.85 E-7}{3.00}$ \\
\hline $1 E-2$ & $8.93 E-3$ & $\frac{1.22 E-3}{2.87}$ & $\frac{1.54 E-4}{2.99}$ & $\frac{1.96 E-5}{2.97}$ & $\frac{2.44 E-6}{3.01}$ & $\frac{3.05 E-7}{3.00}$ \\
\hline $1 E-3$ & $9.10 E-3$ & $\frac{1.25 E-3}{2.86}$ & $\frac{1.59 E-4}{2.97}$ & $\frac{2.02 E-5}{2.98}$ & $\frac{2.53 E-6}{3.00}$ & $\frac{3.14 E-7}{3.00}$ \\
\hline $1 E-4$ & $9.11 E-3$ & $\frac{1.26 E-3}{2.85}$ & $\frac{1.59 E-4}{2.99}$ & $\frac{2.03 E-5}{2.97}$ & $\frac{2.53 E-6}{3.00}$ & $\frac{3.13 E-7}{3.01}$ \\
\hline $1 E-5$ & $\underline{9.11 E-3}$ & $\frac{1.26 E-3}{2.86}$ & $\frac{1.59 E-4}{2.99}$ & $\frac{2.03 E-5}{2.97}$ & $\frac{2.53 E-6}{3.00}$ & $\frac{3.09 E-7}{3.03}$ \\
\hline $1 E-6$ & $9.12 E-3$ & $\frac{1.26 E-3}{2.86}$ & $\frac{1.60 E-4}{2.98}$ & $\frac{2.04 E-5}{2.97}$ & $\frac{2.62 E-6}{2.96}$ & $\frac{3.27 E-7}{3.00}$ \\
\hline
\end{tabular}

\section{ACKNOWLEDGMENTS}

The authors would like to express their deep gratitude to the editor and the referee for their useful suggestions on improving the readability of the paper. Thanks are due to Yu. Rojeck; numerical results were obtained with a program based on a code written by him.

\section{REFERENCES}

1. C. de Boor and B. Swartz, Collocation at Gaussian points, SIAM J. Numer. Anal. 10 (1973), 582-606. MR 51:9528

2. J. Cerutti, Collocation for systems of ordinary differential equations, Comp. Sci. Tech. Rep. 230. Univ. Wisconsin-Madison, 1974.

3. U. Ascher, J. Christiansen, and R. Russell, A collocation solver for mixed order systems of boundary value problems, Math Comp. 33 (1979), 659-679. MR 80b:65108

4. F. Natterer, Uniform convergence of Galerkin's method for splines on highly nonuniform meshes, Math Comp. 31 (1977), 457-468. MR 55:6870

5. P. Ciarlet, The Finite Element Method for Elliptic Problems, North-Holland, Amsterdam, 1978. MR 58:25001

6. V. Thomée, Galerkin Finite Element Methods for Parabolic Problems, Lecture Notes in Math., vol. 1054, Springer-Verlag, Berlin, 1984. MR 86k:65006

7. I. A. Blatov and V. V. Strygin, Convergence of the Galerkin method for nonlinear two-point singularly perturbed boundary value problems in the space $C[a, b]$, Zh. Vychisl. Mat. i Mat. Fiz. 25 (1985), 1001-1008; English transl., USSR Comput. Math. and Math. Phys. 25 (1985), no. 4, 25-30. MR 87b:65102

8. Convergence of the spline collocation method on optimal grids for singularly perturbed boundary value problems, Differentsial'nye Uravnenia 24 (1988), 1977-1987; English transl., Differential Equations 24 (1988), 1330-1338. MR 90f:65128

9. _ The spline collocation method on adaptive meshes for singularly perturbed boundary value problems, Soviet Math. Dokl. 39, (1989), 136-139. MR 90e:65112 
10. I. A. Blatov, The projective method for singularly perturbed boundary value problems, Zh. Vychisl. Mat. i Mat. Fiz. 30 (1990), 1031-1044; English transl. in USSR Comput. Math. and Math. Phys. 30 (1990). MR 91h:65123

11. V. V. Strygin and V. V. Sirunjan, The Galerkin method for singularly perturbed boundary value problem on adaptive meshes, Sibirsk. Mat. Zh. 31 (1990), no. 5, 138-148; English transl., Siberian Math. J. 31 (1990), 817-826. MR 92a:65235

12. U. Ascher and R. Weiss, Collocation for singular perturbation problems. I: First order systems with constant coefficients, SIAM J. Numer. Anal. 20 (1983), 537-557. MR 85a:65113

13. Collocation for singular perturbation problems. II: Linear first order systems without turning pints, Math. Comp. 43 (1984), 157-187. MR 86g:65138a

14. C. Ringhofer, On collocation schemes for quasilinear singularly perturbed boundary value problems, SIAM J. Numer. Anal. 21 (1984), 864-882. MR 86j:65096

15. J. A. Nitsche, $L_{\infty}$-convergence of finite element approximations, Mathematical Aspects of Finite Element Methods, Lecture Notes in Math., Vol. 606, Springer-Verlag, Berlin, 1977, pp. 261-274. MR 58:8351

16. F. Natterer, Über die punktwiese Konvergenz finiter Elemente, Numer. Math. 25 (1975), 67-77. MR 57:14514

17. R. Scott, Optimal $L^{\infty}$ estimates for the finite element method on irregular meshes, Math. Comp. 30 (1976), 681-697. MR 55:9560

18. A. H. Schatz and L. B. Wahlbin, On the finite element method for singularly perturbed reaction-diffusion problems in two and one dimensions, Math. Comp. 40 (1983), 47-89. MR 84c: 65137

19. A. B. Vasil'eva, Asymptotic behavior of solutions of certain problems for ordinary nonlinear differential equations with a small parameter multiplying the highest derivatives, Uspekhi Mat. Nauk 18 (1963), no. 3, 15-86; English transl., Russian Math. Surveys 18 (1963), no. 3, 13-84. MR 28:1363.

20. N. S. Bakhvalov, On optimization of the methods for solving boundary value problems in the presence of a boundary layer, Zh. Vychisl. Mat. i Mat. Fiz. 9 (1969), 841-859; English transl. in USSR Comput. Math. and Math. Phys. 9 (1969). MR 40:8273

21. C. de Boor, A Practical Guide to Splines, Appl. Math. Sci., vol. 27, Springer-Verlag, Berlin, 1978. MR 80a:65027

22. I. A. Blatov and V. V. Strygin, Order-sharp estimates in the Galerkin finite element method for singularly perturbed boundary value problems, Russian Acad. Sci. Dokl. Math. 47 (1993), 93-96. MR 94g:65088

23. R. Weiss, An analysis of the box and trapezoidal schemes for linear singularly perturbed boundary value problems, Math. Comp. 42 (1984), 41-67. MR 86b:65085

Department of Applied Mathematics and Mechanics, Voronezh State University, UniVERsitetskaya Pl.1, Voronezh, Russia, 394693

E-mail address: blatov@kvm.vsu.ru

E-mail address: strygin@kvm.vsu.ru 